\title{
Cholecystokinin-Expressing Interneurons of the Medial Prefrontal Cortex Mediate Working Memory Retrieval
}

\author{
Robin Nguyen, ${ }^{1 \star}$ Sridevi Venkatesan,,${ }^{3 \star}$ Mary Binko, ${ }^{3}$ Jee Yoon Bang,,${ }^{2}$ Janine D. Cajanding, ${ }^{2}$ Chloe Briggs, ${ }^{1}$ \\ Derya Sargin, ${ }^{3}$ Itaru Imayoshi, ${ }^{4}$ Evelyn K. Lambe, ${ }^{3,5,6}$ and ${ }^{-}$Jun Chul Kim ${ }^{1,2}$ \\ ${ }^{1}$ Psychology, Faculty of Arts and Science, University of Toronto, Toronto, Ontario M5S 3G3, Canada, ${ }^{2}$ Cell and Systems Biology, Faculty of Arts and Science, \\ University of Toronto, Toronto, Ontario M5S 3G3, Canada, ${ }^{3}$ Physiology, Faculty of Medicine, University of Toronto, Toronto, Ontario M5S 1A8, Canada, \\ ${ }^{4}$ Institute for Frontier Life and Medical Sciences, Kyoto University, Shogoin-Kawahara 53, Sakyo-ku, Kyoto 606-8507, Japan, ${ }^{5}$ OBGYN, Faculty of Medicine, \\ University of Toronto, Toronto, Ontario M5S 1A8, Canada, and 'Psychiatry, Faculty of Medicine, University of Toronto, Toronto, Ontario M5S 1A8, Canada
}

Distinct components of working memory are coordinated by different classes of inhibitory interneurons in the PFC, but the role of cholecystokinin (CCK)-positive interneurons remains enigmatic. In humans, this major population of interneurons shows histological abnormalities in schizophrenia, an illness in which deficient working memory is a core defining symptom and the best predictor of long-term functional outcome. Yet, CCK interneurons as a molecularly distinct class have proved intractable to examination by typical molecular methods due to widespread expression of CCK in the pyramidal neuron population. Using an intersectional approach in mice of both sexes, we have succeeded in labeling, interrogating, and manipulating CCK interneurons in the mPFC. Here, we describe the anatomical distribution, electrophysiological properties, and postsynaptic connectivity of CCK interneurons, and evaluate their role in cognition. We found that CCK interneurons comprise a larger proportion of the mPFC interneurons compared with parvalbumin interneurons, targeting a wide range of neuronal subtypes with a distinct connectivity pattern. Phase-specific optogenetic inhibition revealed that CCK, but not parvalbumin, interneurons play a critical role in the retrieval of working memory. These findings shine new light on the relationship between cortical CCK interneurons and cognition and offer a new set of tools to investigate interneuron dysfunction and cognitive impairments associated with schizophrenia.

Key words: CCK; GABA; interneuron; mPFC; optogenetics; working memory

\section{Significance Statement}

Cholecystokinin-expressing interneurons outnumber other interneuron populations in key brain areas involved in cognition and memory, including the mPFC. However, they have proved intractable to examination as experimental techniques have lacked the necessary selectivity. To the best of our knowledge, the present study is the first to report detailed properties of cortical cholecystokinin interneurons, revealing their anatomical organization, electrophysiological properties, postsynaptic connectivity, and behavioral function in working memory.

\section{Introduction}

Cognitive processing in cortical networks relies on microcircuit interactions between principle cells and a diverse population of

\footnotetext{
Received Aug. 7, 2019; revised Jan. 20, 2020; accepted Jan. 23, 2020.

Author contributions: R.N., S.V., M.B., J.Y.B., J.D.C., D.S., E.K.L., and J.C.K. designed research; R.N., S.V., M.B., J.Y.B., J.D.C., C.B., and D.S. performed research; R.N., S.V., M.B., D.S., and E.K.L. analyzed data; R.N., S.V., M.B., E.K.L., and J.C.K. wrote the first draft of the paper; R.N., S.V., M.B., E.K.L., and J.C.K. edited the paper; R.N., S.V., E.K.L., and J.C.K. wrote the paper; I.I. contributed unpublished reagents/analytic tools.

This work was supported by Ontario Mental Health Foundation to R.N., Ontario Graduate Scholarship to J.Y.B., Canada Research Chair in Developmental Cortical Physiology and Canadian Institutes of Health Research MOP 89825 to E.K.L., Natural Sciences and Engineering Research Council Discovery MOP 491009 and Canadian Institutes of Health Research MOP 496401 to J.C.K., Ontario Graduate Scholarship to M.B., and University of Toronto Mary H. Beatty Fellowship to S.V.

The authors declare no competing financial interests.

*R.N. and S.V. contributed equally to this work.
}

GABAergic interneurons. Inhibition provided by interneurons coordinates pyramidal cell ensembles and oscillatory activity both spatially and temporally (Klausberger and Somogyi, 2008; Taniguchi, 2014), allowing for meaningful neural activity patterns for cognition (Goldman-Rakic, 1996; Isaacson and Scanziani, 2011). The wide range of GABAergic interneurons have been broadly grouped into subclasses based on distinct combinations of features, such as by their molecular expression profiles, electrophysiological properties, developmental origins, and synaptic targeting of particular subcellular compartments (Kepecs and Fishell, 2014; Lim et al., 2018). 
Cholecystokinin (CCK) interneurons are a subclass of interneurons found to be highly abundant throughout the neocortex and hippocampus (Whissell et al., 2015). While being a relatively heterogeneous population, several common features have been described (Armstrong and Soltesz, 2012; Savanthrapadian et al., 2014; Pelkey et al., 2017). A majority of CCK interneurons are basket cells (Cope et al., 2002; Pawelzik et al., 2002; Freund, 2003; Bezaire and Soltesz, 2013), which densely innervate the cell soma, proximal dendrites, and axon initial segment of pyramidal cells (Halasy et al., 1996; Megías et al., 2001). CCK interneurons largely display regular-spiking (RS) activity (Kawaguchi and Kubota, 1998; Pawelzik et al., 2002) and exert strong rapid feedforward inhibition onto pyramidal cells (Basu et al., 2013). This powerful postsynaptic inhibition is also long-lasting in nature in accordance with their asynchronous GABA release (Hefft and Jonas, 2005; Daw et al., 2009). Moreover, CCK interneurons express several neuromodulatory receptors, such as those for 5HT3a, nicotinic and muscarinic acetylcholine receptors, and the endocannabinoid CB1 receptor (Cea-del Rio et al., 2012; Keimpema et al., 2012), implicating them in behavioral state-dependent processing (Freund, 2003; Wester and McBain, 2014). In particular, high expression of presynaptic CB1 receptors suggests the role of CCK interneurons in synaptic plasticity changes underlying learning and memory (Katona et al., 1999; Bodor et al., 2005; Dudok et al., 2015). Both short-term depolarization-induced suppression of inhibition and inhibitory longterm depression reduce the feedforward inhibition provided by CCK interneurons (Wilson and Nicoll, 2001; Lee et al., 2011; Basu et al., 2013, 2016). The role of CB1 receptors in synaptic plasticity mechanisms is also consistent with the amnestic effects of exogenous cannabinoids, particularly during working memory performance (Ranganathan and D'Souza, 2006).

Working memory is a short-term multistage process involving information encoding, the maintenance of internal representations, and the retrieval and usage of such representations to guide goal-directed behavior (Eriksson et al., 2015). The mPFC is a key substrate for working memory (Curtis and D'Esposito, 2004; Sreenivasan et al., 2014) and displays behavioral correlates, such as sustained population activity and sequential neuronal firing during working memory maintenance (Fuster and Alexander, 1971; Fujisawa et al., 2008; Liu et al., 2014), as well as task phase-specific oscillations and long-range communication with structures supporting memory and behavioral execution (Jones and Wilson, 2005; Ito et al., 2015; Spellman et al., 2015; Lundqvist et al., 2016; Bolkan et al., 2017). These activity signatures of working memory in the mPFC rely on tightly controlled network excitation and inhibition (Carter and Wang, 2007).

The involvement of mPFC CCK interneurons in working memory is indirectly evidenced by the impairments observed following local mPFC application of CB1 receptor agonists (De Melo et al., 2005; Rodrigues et al., 2011). Moreover, adolescent cannabinoid use is associated with the increased risk of developing schizophrenia (Moore et al., 2007), a condition in which working memory impairments are a core deficit (Forbes et al., 2009; Gold et al., 2010; Anticevic et al., 2011). Postmortem studies in schizophrenia also show dysfunctional PFC GABAergic interneurons, including CCK interneurons (Eggan et al., 2008; Hashimoto et al., 2008; Curley and Lewis, 2012; Fung et al., 2014; Marsman et al., 2014). However, cognitive deficits in schizophrenia have primarily been attributed to the disruption of PFC parvalbumin (PV) interneurons (Lewis, 2012), the other major subclass of basket cells (Pawelzik et al., 2002; Baude et al., 2007; Freund and Katona, 2007; Jiang et al., 2015).
Cortical PV interneurons have been extensively studied over the last decade in a wide variety of cognitive processes (Hu et al., 2014; Pinto and Dan, 2015; Canetta et al., 2016; H. Kim et al., 2016; Abbas et al., 2018) and exhibit task-related firing during spatial and nonspatial working memory (D. Kim et al., 2016; Lagler et al., 2016; Kamigaki and Dan, 2017). In contrast, challenges to the selective genetic labeling of CCK interneurons have left this population relatively underinvestigated (Bartos and Elgueta, 2012). In this study, we overcame the technical obstacles of studying CCK interneurons in isolation and investigated their anatomical distribution, electrophysiological properties, and postsynaptic connectivity in the mPFC. With in vivo optogenetic silencing, we evaluated the behavioral role of CCK interneurons in working memory. We found that CCK interneurons comprised a large proportion of the mPFC GABAergic interneuron population, targeting a wide range of neuronal subtypes. At the behavioral level, CCK interneuron activity was found to be required specifically during the retrieval of working memory. These characteristics of CCK interneurons provide a mechanistic understanding of the devastating consequences their disruption may have for working memory and executive functioning in neuropsychiatric conditions.

\section{Materials and Methods}

Animals. Triple-transgenic CCK-Cre;Dlx5/6-FLPe;RC::FrePe mice (termed CCK-FrePe mice) and PV-Cre;Dlx5/6-FLPe;RC::FrePe mice (termed PVFrePe mice) were generated as follows: homozygous RC::FrePe mice (Bang et al., 2012) were crossed with Dlx5/Dlx6-FLPe mice [Tg(mI56iFLPe)39Fsh/J,JAX\#010815] to generate double-transgenic Dlx5/6-FLPe; $\mathrm{RC}::$ FrePe mice, which were then crossed with either homozygous CCKires-Cre mice [B6N.Cg-Ccktm1.1(cre)Zjh/J, JAX\#019021] or PV-ires-Cre mice [B6;129P2-Pvalbtm1(cre)Arbr/J, JAX\#008069]. Triple-transgenic CCK-Cre;Dlx5/6-FLPe;RC::PFArchT mice (termed CCK-ArchT mice) were generated as follows: Dlx5/Dlx6-FLPe mice were crossed with homozygous CCK-ires-Cre mice to generate double-transgenic Dlx5/6FLPe;CCK-Cre mice, which were then crossed with RC::PFArchT mice (obtained from the laboratory of Dr. Itaru Imayoshi). Mice were group housed with ad libitum access to food and water in a temperaturecontrolled room on a $12 \mathrm{~h}$ light/dark cycle. Experimental procedures were in accordance with the guidelines of the Canadian Council on Animal Care and the local Animal Care Committee at the University of Toronto.

Drugs. For slice electrophysiology experiments, bicuculline (Tocris Bioscience, $10 \mu \mathrm{M}$ ) was used to block GABA-A receptors and CGP52432 (Tocris Bioscience, $1 \mu \mathrm{M}$ ) was used to block GABA-B receptors. CNQX (Alomone Labs, $20 \mu \mathrm{M}$ ) and APV (Alomone Labs, $50 \mu \mathrm{M}$ ) were used to block AMPA receptors.

Stereotaxic surgery. Stereotaxic surgery was performed on 3-monthold male mice for behavioral testing and histology experiments, and male and female mice aged 3-6 months for electrophysiological recordings. Mice were anesthetized with isoflurane and mounted onto a stereotaxic frame. For viral delivery of opsins and reporters, PV-Cre mice were injected with an adeno-associated vector (AAV) containing Cre-dependent Archaerhodopsin (ArchT) (AAV5-EF1 $\alpha$-DIO-eArch3.0-EYFP, UNC Vector Core, $4 \times 10^{12}$ particles/ml) or EYFP (AAV5-EF1 $\alpha$-DIO-EYFP, UNC Vector Core, $6 \times 10^{12}$ particles $/ \mathrm{ml}$ ), and CCK-Cre mice received an injection of AAV containing Cre-dependent Channelrhodopsin (ChR2) under the control of the Dlx5/6 enhancer (AAV1-Dlx5/6-DIO-ChR2-EGFP, Vigene Biosciences, custom production, $2 \times 10^{13}$ particles $/ \mathrm{ml}$ ).

All viral infusions were performed bilaterally into the mPFC using the following coordinates: AP $1.90 \mathrm{~mm}, \mathrm{DV}-2.30, \mathrm{ML} \pm 0.50 \mathrm{~mm}$. A volume of $0.3 \mu \mathrm{l}$ was delivered via an internal cannula (33 gauge, Plastics One) connected by Tygon tubing to a $10 \mu \mathrm{l}$ Hamilton syringe. The internal cannula was left in place for $10 \mathrm{~min}$ after infusion to prevent solution backflow. Viral expression was restricted to the cingulate and prelimbic cortex, which span $\sim 1.5 \mathrm{~mm}$ rostrocaudally and $1.5 \mathrm{~mm}$ in depth.

In both PV-ArchT and CCK-ArchT mice, optic fiber probes were implanted bilaterally above the mPFC (AP $1.90 \mathrm{~mm}$, DV -1.75, ML 
Table 1. Electrophysiological properties of pyramidal neurons assessed for postsynaptic responses to light excitation of CCK-ChR2 interneurons ${ }^{a}$

\begin{tabular}{lcc}
\hline & RS $(n=14)$ & BS $(n=9)$ \\
\hline Membrane potential $(\mathrm{mV})$ & $-84 \pm 2$ & $-84 \pm 1$ \\
Input resistance $(\mathrm{m} \Omega)$ & $207 \pm 24$ & $193 \pm 11$ \\
Capacitance $(\mathrm{pF})$ & $57 \pm 3$ & $82 \pm 5^{* * * *}$ \\
Spike threshold $(\mathrm{mV})$ & $-50 \pm 1$ & $-49 \pm 1$ \\
Spike amplitude $(\mathrm{mV})$ & $69 \pm 3$ & $67 \pm 5$ \\
\hline
\end{tabular}

${ }^{a}$ Pyramidal neurons recorded to test for postsynaptic responses to CCK neurons could be classified as either Regular Spiking (RS) or Burst Spiking (BS). These two classes did not differ in their electrophysiological properties, including the resting membrane potential, input resistance, action potential threshold, and spike amplitude. However, they differ significantly in their membrane capacitance (unpaired $t$ test: $\left.t_{(21)}=4.6\right)$. Data are mean \pm SEM.

****p $<0.0001$.

$\pm 0.75 \mathrm{~mm})$ at a $10^{\circ}$ angle away from the midline. The optic fiber probes were secured to the skull using dental cement (RelyX Unicem; 3M). After surgery, mice were individually housed and allowed 1 week to recover before beginning behavioral experiments. Optogenetic manipulations were conducted a minimum of 4 weeks after viral infusion to allow for adequate opsin expression.

Electrophysiology. Electrophysiology was performed on adult male and female mice (3-12 months); no age and sex differences were observed. Animals were anesthetized with an intraperitoneal injection of chloral hydrate $(400 \mathrm{mg} / \mathrm{kg})$ and then decapitated. The brain was rapidly extracted in ice-cold sucrose aCSF (254 mm sucrose, $10 \mathrm{~mm}$ D-glucose, 26 $\mathrm{mm} \mathrm{NaHCO}_{3}, 2 \mathrm{~mm} \mathrm{CaCl}_{2}, 2 \mathrm{~mm} \mathrm{MgSO}_{4}, 3 \mathrm{~mm} \mathrm{KCl}$, and $1.25 \mathrm{~mm}$ $\mathrm{NaH}_{2} \mathrm{PO}_{4}$ ); 400- $\mu$ m-thick slices of the PFC (bregma 2.4-1.1) were obtained on a Dosaka linear slicer (SciMedia). Slices were allowed to recover for $2 \mathrm{~h}$ in oxygenated $\left(95 \% \mathrm{O}_{2} / 5 \% \mathrm{CO}_{2}\right)$ aCSF $(128 \mathrm{~mm} \mathrm{NaCl}$ instead of sucrose, otherwise as above aCSF) at $30^{\circ} \mathrm{C}$ before being used for electrophysiology.

For whole-cell patch-clamp electrophysiology, brain slices were transferred to a chamber mounted on the stage of a BX51WI microscope (Olympus) and constantly perfused with oxygenated aCSF at $30^{\circ} \mathrm{C}$. The recording electrodes $(2-4 \mathrm{M} \Omega$ ) were filled with patch solution composed of $120 \mathrm{~mm}$ potassium gluconate, $5 \mathrm{~mm} \mathrm{KCl}, 10 \mathrm{~mm} \mathrm{HEPES}, 2 \mathrm{~mm} \mathrm{MgCl}_{2}$, $4 \mathrm{~mm}$ K2-ATP, $0.4 \mathrm{~mm} \mathrm{Na2-GTP}$, and $10 \mathrm{~mm}$ sodium phosphocreatine, $\mathrm{pH}$ adjusted to 7.3 using $\mathrm{KOH}$. Data were acquired with a Multiclamp $700 \mathrm{~B}$ or an Axopatch $200 \mathrm{~B}$ amplifier at $20 \mathrm{kHz}$ with Digidata $1440 \mathrm{~A}$ and pClamp 10.7 acquisition software (Molecular Devices). All recordings were compensated for the liquid junction potential $(14 \mathrm{mV})$. Identification and optogenetic stimulation of labeled CCK interneurons were achieved using an LED (Thorlabs, $473 \mathrm{~nm}, 2 \mathrm{~mW}$ through the $60 \times$ lens, $5 \mathrm{~ms}$ light pulses) to excite ChR2 or using an X-Cite (Excelitas) to visualize CCK FrePe and CCK-ArchT-GFP interneurons (467-498 nm excitation filter) and to activate ArchT (532-554 nm excitation filter). For characterizing intrinsic properties and input-output characteristics, neuronal responses were recorded to current steps delivered from rest. For recording light-evoked postsynaptic responses in voltage clamp, cells were held at $-60 \mathrm{mV}$ to permit visualization of either inhibitory or excitatory responses. For identifying postsynaptic targets of CCK interneurons, both pyramidal and interneurons were patched based on morphology, and the different types were distinguished by their firing patterns to current injection. To determine the effect of CCK interneuron stimulation on postsynaptic neuron firing, we injected a step current to elicit firing in the postsynaptic neuron and then compared the average firing rate in the presence and absence of optogenetic stimulation of CCK interneurons over 5 trials.

The patching of potential postsynaptic partner neurons was guided by IR-DIC morphology and their identification informed by electrophysiological parameters. Neuronal properties of these pyramidal cell and interneuron populations are illustrated in Tables 1 and 2, respectively. Pyramidal neurons were identified and patched on the basis of morphology and divided into two subtypes based on their electrophysiological signatures to depolarizing steps in current clamp. Burst spiking (BS) pyramidal neurons were distinguishable by the presence of a burst of AP firing at the beginning of the current step. Regular Spiking (RS) pyramidal neurons lacked bursts and exhibit spike frequency accommodation.
Table 2. Electrophysiological properties of interneurons assessed for postsynaptic responses to light excitation of CCK-ChR2 interneurons ${ }^{a}$

\begin{tabular}{lccc}
\hline & $\mathrm{LT}(n=16)$ & $\mathrm{RS}(n=14)$ & $\mathrm{FS}(n=18)$ \\
\hline Membrane potential $(\mathrm{mV})$ & $-78 \pm 1$ & $-79 \pm 2$ & $-80 \pm 2$ \\
Input resistance $(\mathrm{m} \Omega)$ & $533 \pm 40^{a a, b b}$ & $259 \pm 26^{a a, c}$ & $144 \pm 12^{b b, c}$ \\
Capacitance $(\mathrm{pF})$ & $41 \pm 2^{a a}$ & $65 \pm 6^{a a, c c}$ & $41 \pm 2^{c c}$ \\
Spike threshold $(\mathrm{mV})$ & $-55 \pm 1^{a a, b b}$ & $-50 \pm 1^{a a}$ & $-49 \pm 1^{b b}$ \\
Spike amplitude $(\mathrm{mV})$ & $74 \pm 3^{b b}$ & $66 \pm 3$ & $58 \pm 2^{b b}$ \\
\hline
\end{tabular}

${ }^{a}$ Interneurons recorded to test for postsynaptic responses to CCK neurons could be segregated into one of three electrophysiological classifications based on their spiking characteristics: Low Threshold (LT), Regular Spiking (RS) or Fast Spiking (FS). These three different classes of interneurons did not differ statistically in the resting membrane potential, but one-way ANOVA found that there were significant group differences on all the other measures, including input resistance $\left(F_{(2,45)}=53.7, p<0.0001\right)$, capacitance $\left(F_{(2,45)}=13.7, p<0.0001\right)$, spike threshold $\left(F_{(2,4)}=15.4, p<0.0001\right)$, and spike amplitude $\left(F_{(2,45)}=9, p<0.01\right)$. Post hoc comparison results show that the low threshold group differed significantly from the FS group on input resistance, spike threshold, and spike amplitude and differed from the RS group on input resistance, capacitance, and spike threshold. The RS interneurons differed significantly from the FS group in terms of input resistance and capacitance. Data are mean \pm SEM.

Comparisons between interneuron groups are denoted by the pairs of superscript letters: Superscript $a$ denotes comparison between low threshold and RS interneurons. Superscript $b$ is between low threshold and FS interneurons. Superscript $c$ is between RS and FS interneurons. ${ }^{a, b, c} p<0.05$; ${ }^{a a, b b, c c} p<0.01$; Tukey's post hoc test.

Interneurons were selected on the basis of morphology and their subtype identified on the basis of electrophysiological characterization. Low threshold firing interneurons were characterized by their low rheobase; that is, they required very small current injections $(<20-30 \mathrm{pA})$ to spike. RS interneurons were characterized by their sharp AHPs and spike frequency accommodation. Fast-spiking (FS) interneurons showed characteristic high-frequency action potential firing.

Since a proportion of interneurons are CCK-positive, it was not surprising that a subset of the interneurons tested for postsynaptic connectivity also expressed ChR2 or were gap junction-coupled to other interneurons that expressed ChR2 (Galarreta and Hestrin, 1999). In this proportion, direct or gap junction-coupled ChR2 photocurrents occurred with submillisecond timing upon light onset (latency: $0.36 \pm 0.08$ ms, $n=10$ ). We examined these traces carefully for evidence of lightevoked IPSCs on top of the ChR2 photocurrent; and where possible, neurons were held at more depolarized potentials to reduce the ChR2 current and confirm apparent presence/absence of light-evoked IPSCs.

Behavioral apparatuses and testing procedures. Handling and food deprivation began 1 week after surgery. Mice were first tested for working memory in an olfactory delayed-nonmatch-to-sample task (DNMS) task. One week after the completion of DNMS testing, mice were trained in a Go/No-go olfactory discrimination task as a control to evaluate changes in nonworking memory processes. A between-subjects design was used for both tasks.

Apparatus. Olfactory DNMS was tested in a gray Plexiglas apparatus composed of three serial compartments separated by sliding doors. The first and second compartments were square in shape with dimensions of $15 \mathrm{~cm} \times 15 \mathrm{~cm} \times 20 \mathrm{~cm}$. The third compartment was a semicircular platform (25 cm radius). Odors were powdered spices (cinnamon or thyme) mixed with Aspen woodchips and were presented in metal wells (3.5 $\mathrm{cm}$ diameter). A single sucrose pellet was placed beneath the woodchips of both wells to prevent the use of the sucrose scent to guide choice behavior. The testing room was illuminated with 150 lux lighting, and background noise was masked with a white noise generator. Animal movements were tracked using ANY-maze (Stoelting).

Habituation. Five days before testing, mice were handled daily for 5 min. During this time, mice were food deprived and maintained at $85 \%$ of their free feeding weight throughout testing. Two days before testing, mice were habituated to the apparatus and to the test handling procedure for $30 \mathrm{~min}$. During these sessions, mice were handled in the same manner as during testing. Mice were trained to dig by burying the sucrose pellet successively deeper. After habituation sessions, sucrose pellets were provided overnight.

Olfactory DNMS procedure. Testing occurred on consecutive days $7 \mathrm{~d}$ a week. Mice were acclimated to the testing room for $5 \mathrm{~min}$ before the beginning of the test. At the start of each trial, one scented well, either cinnamon or thyme (sample odor), was placed in the first compartment, and two scented wells, one of each odor, were placed in the third compartment. Individual mice were placed in the first compartment and 
allowed to retrieve and consume the reward (sample phase), after which they were enclosed in the second compartment for $5 \mathrm{~s}$ (delay phase). After the delay, the partition was removed, and the mouse was allowed to enter the third compartment and dig in one of the two odors (response phase). Digging in the nonmatching odor constituted a correct response and permitted the retrieval of reward. Digging in the matching odor was considered incorrect and the well was immediately removed. Digging behavior was scored as the displacement of woodchips with either the nose or forepaws. The mouse was then placed in the home cage for a 1 min intertrial interval, after which the next trial was conducted as described above.

The sample odor was randomized across trials with no more than 2 consecutive trials for a given odor. In the response compartment, the positions of the matching and nonmatching odors were counterbalanced across trials, such that the nonmatching odor was encountered first in half the trials and second in the other half in a randomized order. On trials in which the nonmatching odor was encountered first, mice were required to immediately dig (hit response), while encountering the matching odor required withholding of digging and moving to dig in the second nonmatching odor (correct rejection response). Withholding digging in the nonmatching odor was scored as a miss response, while digging in the matching odor was scored as a false alarm. The spatial locations of the odors on the platform were also randomized and counterbalanced to limit proactive interference and response bias. Shaping took place over $4 \mathrm{~d}$ with one session of 16 trials each day. After shaping, a total of 36 trials were performed in each daily session until criterion was reached. Criterion was defined as a percent correct performance of either $\geq 70 \%$ on 2 consecutive days or $\geq 80 \%$ on a single day. The percent correct performance was calculated as follows: (number of correct trials over the total number of trials) $\times 100$. The percentage of each response type (hit, correct rejection, miss, false alarm) was calculated as follows: (number of a given response type over the total number of possible trials for that type) $\times 100$.

To test the effect of delay interval length on performance, mice were first trained with a $5 \mathrm{~s}$ delay interval until criterion performance was reached and were then tested with a variable delay interval over $2 \mathrm{~d}$. Delays of 5, 20, and $60 \mathrm{~s}$ were tested in one session, and 15, 25, and $45 \mathrm{~s}$ in the other session, with session counterbalanced between days. Each delay interval was conducted on 12 trials for a total of 36 trials per session and were pseudorandomized such that a given interval did not occur on $>2$ consecutive trials and occurred at least once across 5 trials.

Olfactory DNMS testing with optogenetics. After reaching criterion, mice were tested with light delivery via optic fiber probes. Optic fiber probes were constructed from optical fibers (single mode, $0.35 \mathrm{NA}, 200$ $\mu \mathrm{m}$ diameter; Thorlabs) secured to ceramic ferrules ( $1.25 \mathrm{~mm}$ diameter, $230 \mu \mathrm{m}$ bore; Precision Fiber Products). Ceramic mating sleeves (Precision Fiber Products) connected these implants to optic patch cables $(0.37$ NA; Doric Lenses). For bilateral light delivery, two such cables were connected to a 1-2 splitting optical commutator (Doric Lenses), which was in turn connected to an additional patch cable (0.37 NA; Doric Lenses). This patch cable was coupled to a diode-pumped solid-state laser, emitting green light (532 nm; Laserglow). Laser light was applied continuously at a power intensity of $15 \mathrm{~mW}$ from the optic fiber probe tip during the sample, delay, or response phase of the task. Under these conditions, we estimate that an effective power density for $50 \%$ ArchT activation $\left(7.5 \mathrm{~mW} / \mathrm{mm}^{2}\right)$ was applied at a maximum distance of $\sim 1$ $\mathrm{mm}$ from the optic fiber tip, within the boundaries of the mPFC (Deisseroth Lab, Brain tissue light transmission calculator). Light delivery was controlled via transistor-transistor logic signals, which triggered and deactivated the laser output. Transistor-transistor logic signals were sent based on the animal's center body position in the apparatus using ANYmaze (Stoelting), such that, for a given phase, entry and exit from the corresponding chamber triggered the onset and offset of light, respectively. Light illumination was presented during each phase for 12 trials, for a total of 36 trials in a single session. Sample, delay, and choice light illumination were pseudorandomized across trials such that light delivery in a given phase occurred on no more than 2 consecutive trials and occurred at least once over 5 trials. The percent correct performance for each illumination phase was calculated as follows: (number of correct trials for that illumination phase over total number of trials for that illumination phase) $\times 100$.

Go/No-go olfactory discrimination procedure. Following testing in the olfactory DNMS task, mice were trained to perform a simple Go/No-go olfactory discrimination task in the same apparatus. One of two odors, either cardamom or coriander spice, was paired with the sucrose reward throughout all trials. Testing followed the same procedures as with the DNMS task, except that no sample odor was presented. Mice were placed into the first compartment, then held in the delay compartment for $5 \mathrm{~s}$, after which the partition door was removed, and they entered the response compartment where they were presented with the two odors. Digging in the paired odor constituted a correct response, and mice were allowed to retrieve the reward, whereas digging in the unpaired odor was unrewarded and resulted in termination of the trial. Mice were trained to a criterion performance of $\geq 75 \%$ in one session and then tested with optogenetics to inhibit either PV or CCK interneurons.

Histology. Mice were transcardially perfused with PBS, pH 7.4, followed by $4 \%$ PFA. Brains were extracted and postfixed overnight in $4 \%$ PFA at $4^{\circ} \mathrm{C}$ and then cryoprotected with PBS containing 30\% sucrose. Brains were sectioned coronally at $40 \mu \mathrm{m}$ thickness using a cryostat (Leica Microsystems, CM 1520). For immunostaining, free-floating brain sections were blocked with $5 \%$ normal donkey serum in $0.1 \%$ Triton X-100 in PBS (PBS-T) for $1 \mathrm{~h}$. Sections were then incubated for $48-72 \mathrm{~h}$ at $4^{\circ} \mathrm{C}$ with PBS-T containing a combination of the following primary antibodies: rabbit polyclonal anti-PV antibody (1:1000, Abcam, ab11427), rabbit polyclonal anti-CCK (1:1000, Sigma Millipore, C2581; Frontier Institute, Af350), rabbit polyclonal anti-GABA (1:1000, Sigma Millipore, A2052), rabbit polyclonal anti-vasoactive intestinal peptide (VIP) (1:500, Immunostar, 20077), chicken polyclonal anti-GFP (1: 1000 , Abcam, ab13970), and goat polyclonal anti-mCherry (1:1000, Sicgen, AB0040-200). Primary antibody incubation was followed by incubation for $2 \mathrm{~h}$ at room temperature with PBS-T containing the following secondary antibodies: AlexaFluor-488-conjugated donkey antirabbit (1:1000, Jackson ImmunoResearch Laboratories, 711545152), AlexaFluor-594-conjugated donkey anti-rabbit (1:1000, Jackson ImmunoResearch Laboratories, 715515152), Cy5-conjugated donkey antirabbit (1:1000, Jackson ImmunoResearch Laboratories, 711175152), AlexaFluor-488-conjugated donkey anti-chicken (1:1000, Jackson ImmunoResearch Laboratories, 703545145), and AlexaFluor-594-conjugated donkey anti-goat (1:1000, Jackson ImmunoResearch Laboratories, 705515147).

Statistical analysis. For light-evoked IPSC analysis, parameters were estimated using the template detection option in Clampfit 10.7 (Molecular Devices), and IPSCs were fit as product of exponentials. Parameters, including peak amplitude and onset latency, were averaged over 5 trials (with $10 \mathrm{~s}$ intertrial intervals). Graphs and statistics were obtained using Prism (GraphPad) and MATLAB (The MathWorks). Bar graphs represent mean \pm SEM wherever applicable. Cell counting data were analyzed using mixed ANOVAs with genotype (CCK-FrePe or PV-FrePe) as the between-subjects factor and cortical layer as the within-subjects factor. Post hoc analyses comparing CCK- and PV-FrePe data were performed using planned pairwise comparisons with unpaired Student's $t$ tests, whereas within-subjects multiple comparisons were performed with paired Student's $t$ tests with Bonferroni correction. Behavioral data were analyzed separately for CCK-Cre and PV-Cre groups. Mixed ANOVAs were performed with treatment $\left(\mathrm{ArchT}^{+}\right.$or $\left.\mathrm{ArchT}^{-}\right)$as the betweensubjects factor and test phase as the within-subjects factor. Statistically significant interactions were followed by planned pairwise comparisons using unpaired Student's $t$ test. Statistical analyses were performed using SPSS Statistics version 21 (IBM).

\section{Results}

\section{Intersectional genetic targeting of CCK interneurons}

Due to broad CCK expression in both CCK interneurons and pyramidal neurons (Dimidschstein et al., 2016), CCK interneurons cannot be selectively targeted with conventional single recombinase methods. Consistently, we found that infusion of the AAV vector containing Cre-dependent EYFP into the mPFC of 
A

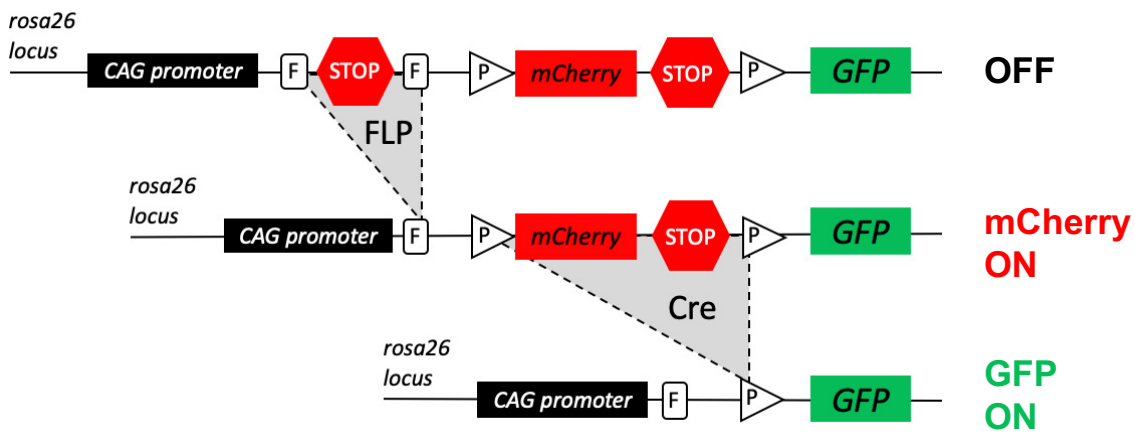

C

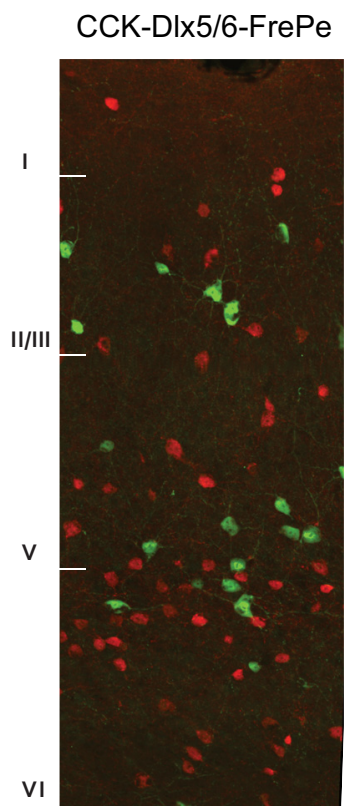

D

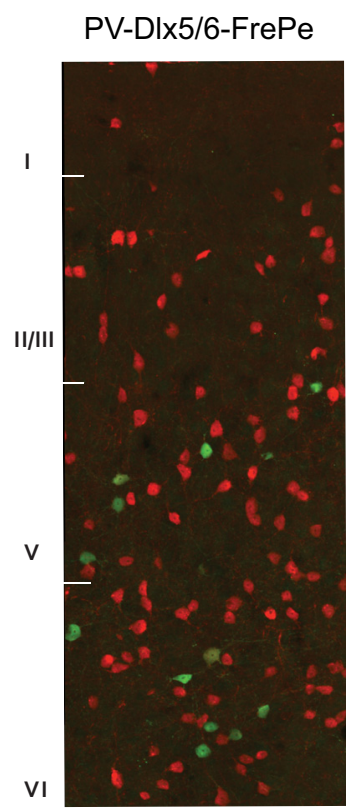

B

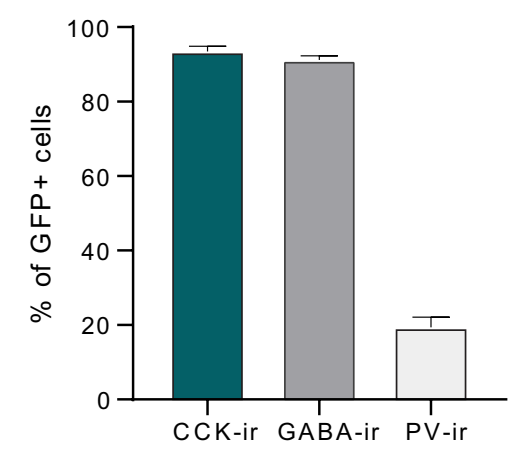

E

aCCK INs $\square \mathrm{PV} I N s$

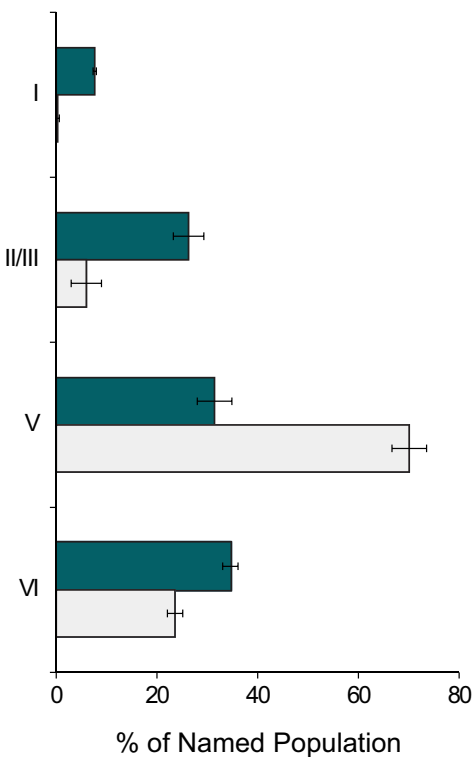

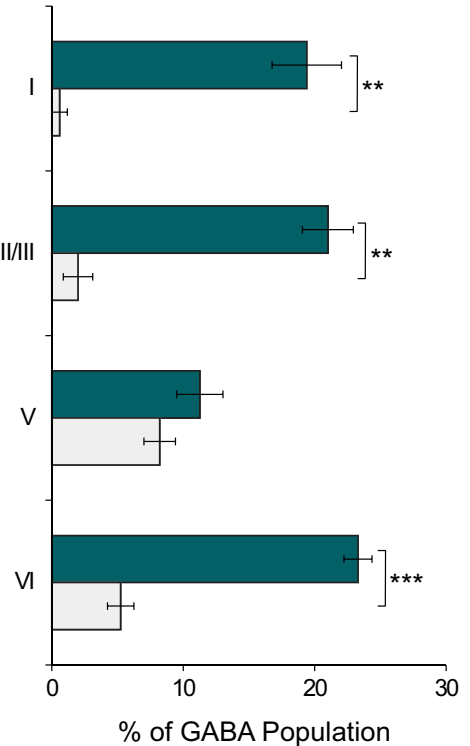

Figure 1. Intersectional genetic labeling and mPFC distribution of CCK and PV interneurons. A, Dual recombinase-responsive reporter allele, RC::FrePe, containing FRT-flanked and loxP-flanked transcriptional stop cassettes. Middle, FLPe-mediated stop cassette removal results in mCherry expression. Bottom, Additional Cre-mediated excisions remove mCherry and the second stop cassette, resulting in GFP expression. The RC::FrePe allele is knocked in to the Gt(ROSA)26Sor(R26) locus with CAG (chicken $\beta$-actin and CMV enhancer) promoter elements. B, Percentage of GFP-labeled cells (i.e., CCK interneurons) that are positive for CCK, GABA, or PV immunoreactivity. $N=3$. Data are mean \pm SEM. C, Representative confocal images of mPFC from CCK-Dlx5/6-FrePe (left) or PV Dlx5/6-FrePe (right) mice. Green represents GFP in CCK or PV interneurons. Red represents mCherry in CCK-negative or PV-negative GABA interneurons. D, Percentage of the total CCK or PV cells found in each layer. $\boldsymbol{E}$, Percentage contribution of $\mathrm{CCK}$ or PV interneurons to the total GABA interneuron population by layer. $N=3 .{ }^{* *} p<0.01,{ }^{* * *} p<0.001$. Data are mean \pm SEM.

CCK-Cre mice resulted in widespread expression of EYFP throughout the targeted area (data not shown). Therefore, we applied a dual recombinase-based intersectional approach (Awatramani et al., 2003; J. C. Kim et al., 2009), using both the Cre/loxP and FLPe/FRT systems, to selectively target CCK interneurons (Whissell et al., 2015). To this end, we crossed the forebrain pan-GABAergic driver Dlx5/6-FLPe (Miyoshi et al., 2010) with the dualrecombinase reporter line RC::FrePe (Engleka et al., 2012). Subsequently, we crossed the double-transgenic offspring (Dlx5/ 6-FLPe, RC::FrePe) with CCK-Cre mice to generate tripletransgenic CCK-FrePe mice (CCK-Cre, Dlx5/6-FLPe, RC:: FrePe). The Cre/FLPe-dependent reporter allele, RC::FrePe, contains two transcriptional stop cassettes (Fig. 1A): FRT sites flank the first stop cassette, whereas loxP sites flank the second cassette and mCherry sequences. FLPe-mediated excision alone, in GABA neurons, results in mCherry expression, while the remaining loxP-flanked stop cassette prevents GFP expression. Thus, in triple-transgenic mice containing all three alleles
(CCK-Cre, Dlx5/6-FLPe, RC::FrePe), FLPe- and Cre-mediated excisions together allow for GFP expression only in CCK interneurons (Fig. $1 A, C$ ). For comparison, the same approach was applied to the PV-Cre mouse line to generate triple-transgenic PV-FrePe mice (PV-Cre, Dlx5/6-FLPe, RC::FrePe).

Consistent with our previous study (Whissell et al., 2015), we found that $93 \%$ and $91 \%$ of GFP-positive cells in the mPFC of CCK-FrePe mice were positive for CCK and GABA immunoreactivity, respectively, and that $78 \%$ of cells positive for both CCK and GABA were GFP-positive, indicating specific and high efficacy labeling of CCK interneurons by the intersectional approach (Fig. $1 B)$, whereas a relatively small number $(\sim 19 \%$ of GFPpositive cells) were positive for PV immunoreactivity (Fig. 1B), which is consistent with previous reports that a small subset of FS PV interneurons express a low level of CCK and represent a molecularly distinct interneuron population that express both CCK and PV (Tricoire et al., 2011; Harris et al., 2018). 
A

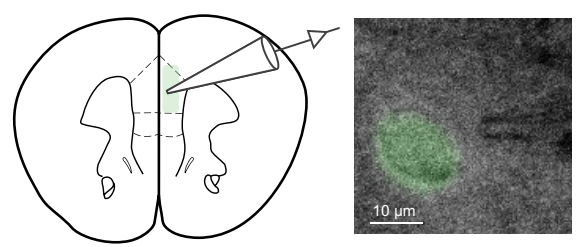

C

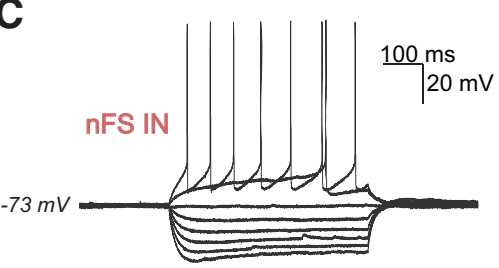

E

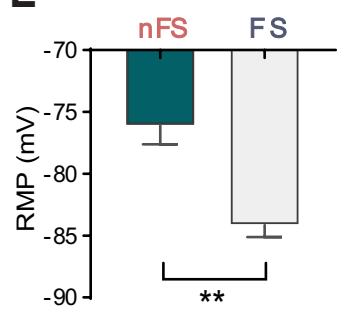

$\mathbf{F}$

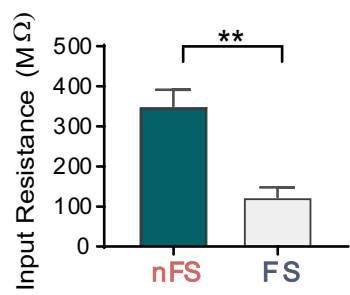

H
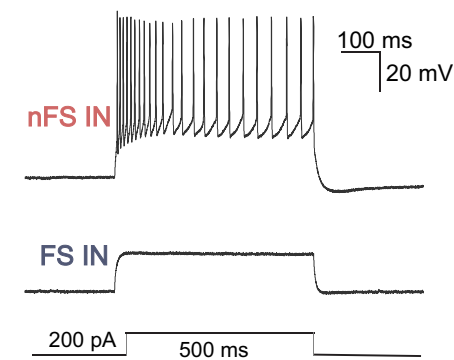

B

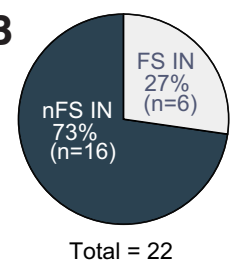

D

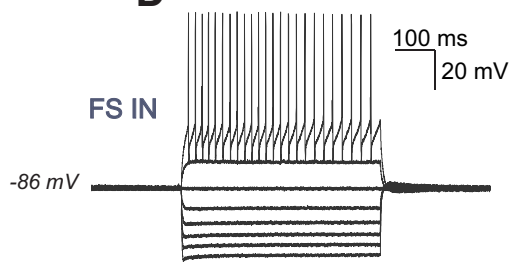

G

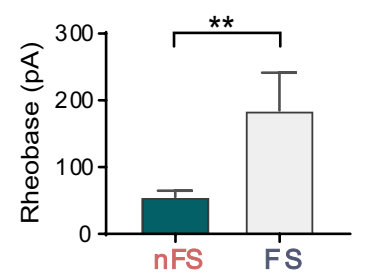

I

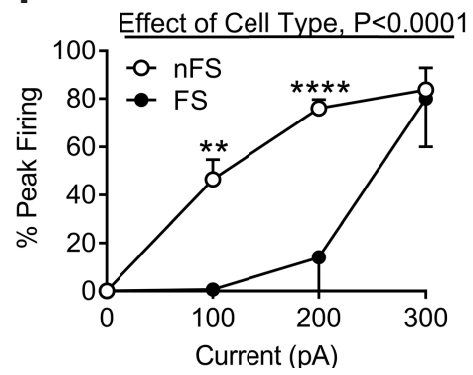

Figure 2. Electrophysiological properties of GFP-positive CCK interneurons from CCK-FrePe mice. $A$, Schematic of the mPFC with the green box representing where whole-cell patch-clamp recordings of GFP-positive CCK interneurons were performed. The IR-DIC image with GFP fluorescence overlay shows an example GFP-positive CCK interneuron. B, Pie chart represents the distribution of GFP-positive CCK non-Fast Spiking (nFS) and Fast-Spiking (FS) interneurons (INs). C, A representative current-clamp trace shows the electrophysiological signature of an $\mathrm{nFS}$ IN in response to $25 \mathrm{pA}$ depolarizing and hyperpolarizing current steps. $\boldsymbol{D}, \mathrm{A}$ representative current-clamp trace shows the electrophysiological signature of an FS IN in response to $150 \mathrm{pA}$ depolarizing and hyperpolarizing current steps. $\boldsymbol{E}$, Bar graph represents a more depolarized resting membrane potential (RMP) in nFS INs than FS INs (unpaired $t$ test, $t_{(20)}=3.2,{ }^{* *} p=0.005$ ). $\boldsymbol{F}$, Bar graph represents a higher input resistance in nFS INs than FS INs (unpaired $t$ test, $\left.t_{(20)}=3.3,{ }^{* *} p=0.004\right)$. G, Bar graph shows that the current required to elicit a single action potential is smaller in nFS INs than FS INs (unpaired $t$ test, $t_{(12)}=4.1,{ }^{* *} p=0.002$ ). $\boldsymbol{H}$, The representative current-clamp traces show the response of an $\mathrm{nFS}$ IN and an FS IN to a $200 \mathrm{pA}$ depolarizing step. The representative traces were chosen to illustrate that FS cells need a much higher current step to start firing. $I$, Graph shows that the percent peak firing is greater in $\mathrm{nFS} \mathrm{INs}(N=14)$ than $\mathrm{FS} \mathrm{INs}(N=5)$ at lower current injections (two-way ANOVA, effect of cell type, $\left.F_{(1,68)}=18.4, p<0.0001\right) .{ }^{* *} p \leq 0.01$ (Bonferroni's post hoc test). ${ }^{* * * *} p \leq$ 0.0001 (Bonferroni's post hoc test). Data are mean \pm SEM.

\section{CCK interneurons are differentially distributed from PV} interneurons in the mPFC

A previous study using the same intersectional approach reported differences in the overall density of CCK and PV interneurons in the mPFC (Whissell et al., 2015). Since functional differences exist between layers of the neocortex (Douglas and Martin, 2004; van Aerde and Feldmeyer, 2015), we determined whether the intersectionally labeled CCK and PV interneurons exhibit differential laminar localization in the mPFC. Cell counting in the prelimbic cortex was performed to determine the distribution of each interneuron type and their relative abundance to the whole GABA interneuron population. The total number of GABA interneu- rons labeled in CCK- and PV-FrePe mice did not significantly differ (unpaired $t$ test, $t_{(4)}=-1.07, p=0.345$ ). The majority of PV interneurons were located in layer 5 (Fig. $1 D ; 70.1 \pm 5.9 \%$ ) and were rarely observed in superficial layers (Fig. $1 D$; layer 1: $0.3 \pm 0.6 \%$, layer 2/3: $6.0 \pm$ $5.2 \%)$. CCK interneurons, on the other hand, were spread more evenly, with comparable levels in layers $2 / 3,5$, and 6 (Fig. 1D; pairwise comparisons with Bonferroni correction, $p>0.05)$. Overall, CCK interneurons comprised a larger proportion of the mPFC GABA interneuron population relative to $\mathrm{PV}$ interneurons (CCK: $16.7 \pm 2.1 \%$, PV: $5.8 \pm 1.9 \%$ unpaired $t$ test, $\left.t_{(4)}=6.80, p<0.01\right)$. This relationship was maintained across cortical layers (Fig. 1E; mixed ANOVA, main effect of group, $F_{(1,4)}=97.63, p<0.001$; main effect of layer, $F_{(3,12)}=5.13, p<$ 0.05 ; phase $\times$ group interaction, $F_{(3,12)}=$ $18.01, p<0.0001)$, with the exception of layer 5 (Fig. $1 E$; unpaired $t$ test, layer $1: t_{(4)}$ $=6.96, p<0.01$, layer $2 / 3, t_{(4)}=8.50, p<$ 0.001 , layer $6: t_{(4)}=4.29, p<0.05$, layer 5: $\left.t_{(4)}=1.43, p=0.225\right)$, in which CCK and PV interneurons comprised a similar proportion of GABA interneurons (CCK: $11.3 \pm 3.1 \%$, PV: $8.2 \pm 2.1 \%)$. Together, these data indicate that CCK and PV interneurons exhibit differences in their abundance and distribution across layers of the mPFC, which supports potential functional differences in their circuit performance and possibly behaviors mediated by the mPFC.

\section{Electrophysiological properties of CCK interneurons}

Next, we characterized electrophysiological properties of CCK interneurons in patch-clamp recordings from $\mathrm{mPFC}$ slices of CCK-FrePe mice. The putative CCK interneurons were identified by GFP fluorescence and patched under visual control using IR-DIC for whole-cell recording (Fig. 2A). Of a total of 22 cells recorded, 16 (73\%) cells displayed non-Fast Spiking (nFS) and 6 cells (27\%) Fast Spiking (FS) activity (Fig. 2B-D). FS cells were clearly distinguishable based on their sudden transition from no spiking to high-frequency, nonaccommodating spiking in response to stronger depolarizing current steps, a feature known as Type II excitability (Prescott, 2014). These cells fit within the category of PV-positive FS interneurons well described in the literature (Kawaguchi and Kubota, 1997, 1998; Kawaguchi and Kondo, 2002). The FS CCK interneurons were able to sustain high firing rates $(>100 \mathrm{~Hz})$ at higher current injections (peak frequency, $196 \pm 19 \mathrm{~Hz}, n=5$ ), whereas stronger depolarizations in a nFS cell failed to further increase firing rates (peak frequency, $40 \pm 6$ $\mathrm{Hz}, n=14$ ). There was no overlap in the firing rates of the two groups of CCK interneurons. The nFS and FS cells also differed 
in other electrophysiological properties: compared with the FS-CCK interneurons, nFS-CCK interneurons had a more depolarized resting membrane potential (Fig. $2 E ;-76 \pm 1 \mathrm{mV}, N=16 \mathrm{vs}-84 \pm 1 \mathrm{mV}$, $N=6$; unpaired $t$ test, $t_{(20)}=3.2, p=$ 0.005 ), a higher input resistance (Fig. $2 F$; $350 \pm 41 \mathrm{M} \Omega, N=16$ vs $124 \pm 24 \mathrm{M} \Omega$, $N=6$; unpaired $t$ test, $t_{(20)}=3.3, p=$ 0.004 ), and a lower minimum current (rheobase) required to evoke an action potential (Fig. $2 G ; 55 \pm 9 \mathrm{pA}, N=11$ vs $185 \pm 56 \mathrm{pA}, N=3$; unpaired $t$ test, $t_{(12)}$ $=4.1, p=0.002)$. Consistently, the output in firing frequency in response to incremental current steps (input-output curve) revealed that nFS-CCK interneurons are more excitable compared with FS-CCK interneurons (Fig. $2 H$; two-way ANOVA, effect of cell type, $F_{(1,68)}=18.4$, $p<0.0001)$. Thus, our data suggest that, based on their electrophysiological properties, CCK interneurons in the $\mathrm{mPFC}$ are divided largely into two distinct subtypes.

\section{Postsynaptic targets of}

\section{CCK interneurons}

Specific synaptic connectivity among excitatory and inhibitory neurons is essential for information processing in cortical circuit operations. To reveal the postsynaptic cell responses evoked by CCK interneuron activity, we virally expressed ChR2 in CCK interneurons using a Cre-responsive AAV vector that drives transgene expression under the control of the Dlx5/6 enhancer (AAV-Dlx5/6-DIO-ChR2) (Miyoshi et al., 2010). Similar to the dual recombinasebased intersectional approach using CCKCre and Dlx5/6-FLPe, the intersectional viral vector restricts transgene expression exclusively in cells where both the CCK promoter and Dlx5/6 enhancer are active (i.e., CCK interneurons). The infusion of AAV-Dlx5/6-DIO-ChR2 into the mPFC of CCK-Cre mice produced robust ChR2 expression across all layers of the $\mathrm{mPFC}$, consistent with the pattern observed in CCK-FrePe mice. Labeled CCK interneurons were identified by their fluorescence and further confirmed by their direct response to optogenetic stimulation (Fig. 3A). As found previously in CCK-FrePe mice, nFS and FS CCK interneurons were identified based on differences in their firing pattern to current injection (Fig. 3B) and electrophysiological properties (Fig. 3C,D).

Non-FS CCK interneurons showed a more depolarized resting membrane potential $(-62.3 \pm 2.1 \mathrm{mV}, N=8)$ compared with FS CCK interneurons $\left(-76.5 \pm 1.6 \mathrm{mV}, N=5\right.$ ) (unpaired $t$ test, $t_{(11)}=$ $4.77, p<0.001)$ and higher input resistance $(229 \pm 20 \mathrm{M} \Omega)$ compared with FS interneurons $\left(149 \pm 17 \mathrm{M} \Omega\right.$ ) (unpaired $t$ test, $t_{(11)}=$ $2.74, p=0.02$ ). Action potentials could be elicited in both neuronal

$\mathbf{E}$

G
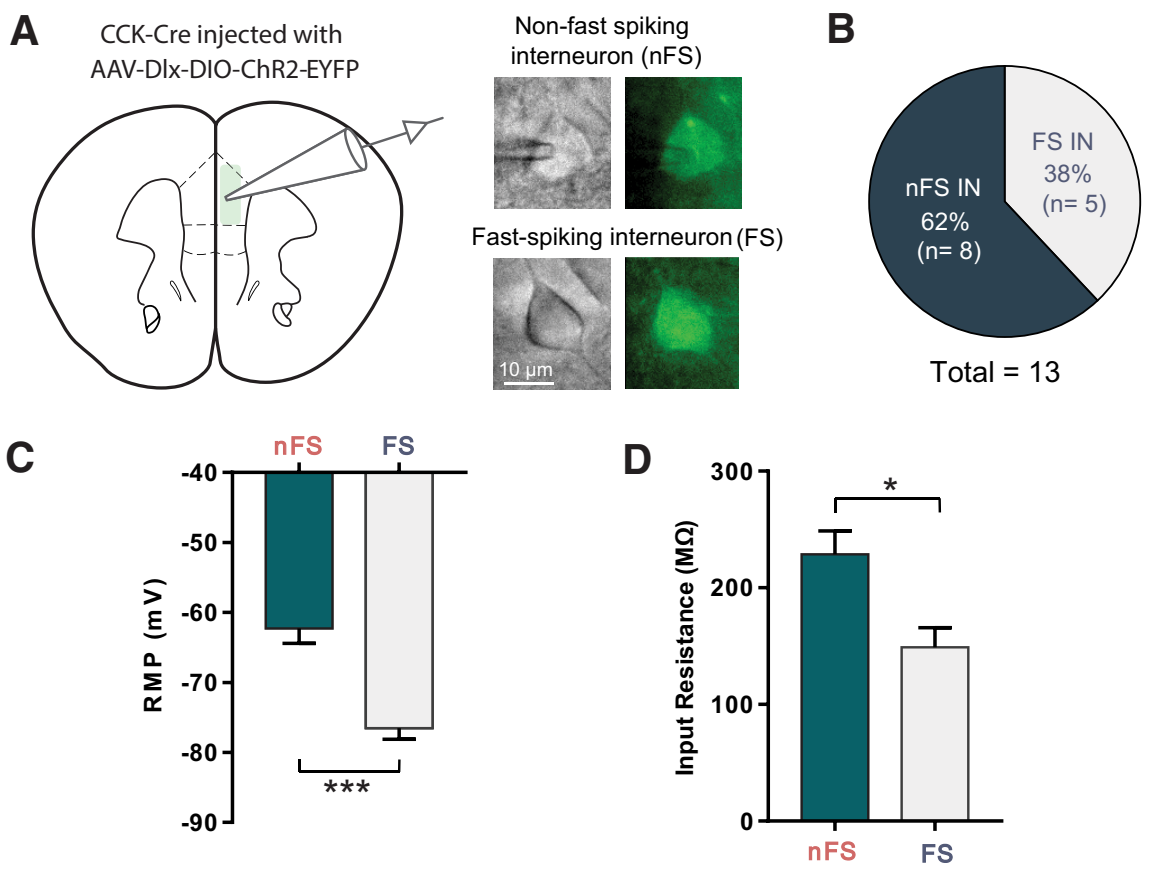

Non-fast spiking interneuron $(\mathrm{nFS})$

$\mathbf{F}$
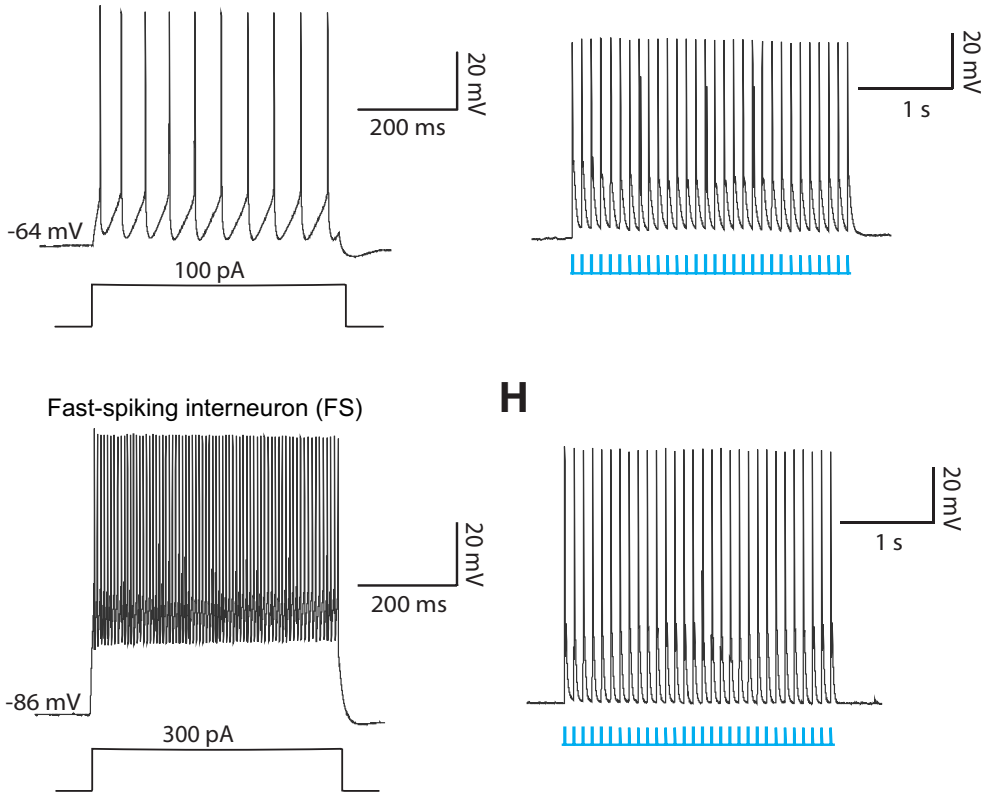

H

3. Characterization of virally transfected ChR2-eYFP expression in CCK GABAergic interneurons. $A$, Schematic showing recording site in PFC. Right, Example non-Fast Spiking (nFS) and Fast Spiking (FS) ChR2-eYFP-expressing interneurons in virally transfected mice. The IR-DIC and fluorescence images are shown together for each neuron. $\boldsymbol{B}$, Pie chart represents the recorded proportions of these two types of $C \mathrm{CK}$ interneurons. $C$, Bar graph shows that $\mathrm{FS}$ and $\mathrm{nFS}$ interneurons differ significantly in their resting membrane potential (RMP). ${ }^{* * *} p<0.001$ (unpaired $t$ test). $\boldsymbol{D}$, Bar graph shows that $\mathrm{FS}$ and $\mathrm{nFS}$ interneurons also differ significantly in their input resistance. ${ }^{*} p<0.05$ (unpaired $t$ test). Example traces showing the sustained action potential firing in a CCK-positive nFS interneuron: $(\boldsymbol{E})$ to a $100 \mathrm{pA}$ step current injection and $(\boldsymbol{F})$ to a $10 \mathrm{~Hz}$ train of light flashes. Example traces for a CCK-positive FS interneuron showing the responses: $(\boldsymbol{G})$ to a $300 \mathrm{pA}$ step current injection and $(\boldsymbol{H})$ to a $10 \mathrm{~Hz}$ light train. The representative current-injection traces for $\mathrm{FS}$ and $\mathrm{nFS}$ interneurons were chosen to illustrate the characteristic high-frequency firing exhibited by FS neurons, which distinguishes them as a group. Data are mean \pm SEM.

types by $5 \mathrm{~ms}$ pulses of blue light delivered at $10 \mathrm{~Hz}$ (Fig. $3 \mathrm{E}, \mathrm{F}$ ). The mean photocurrent elicited did not differ statistically between the FS $(5.1 \pm 1.8 \mathrm{nA}, N=5)$ and the $\mathrm{nFS}$ interneurons $(4.8 \pm 2.7 \mathrm{nA}, N=$ 8) (unpaired $t$ test, $t_{(11)}=0.14, p=0.9$ ).

To identify the postsynaptic targets of CCK interneurons, we patched nearby pyramidal neurons and interneurons (Fig. 4A, B; 
A CCK-Cre mouse injected with AAV-DIX-DIO-ChR2-YFP

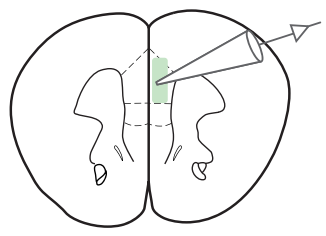

D

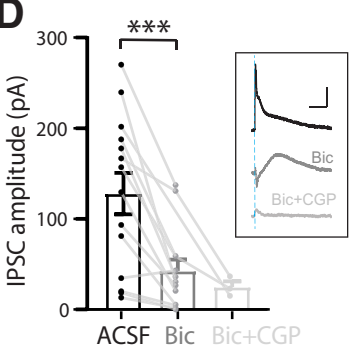

G

1 Burst spiking pyramidal

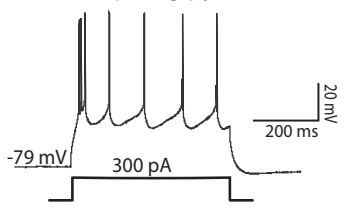

2

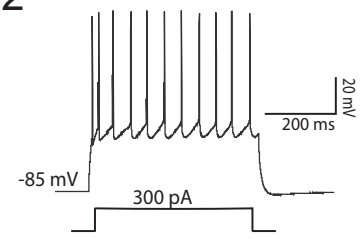

3 Low threshold interneuron

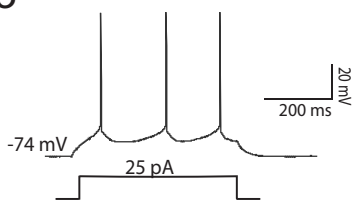

4

4 Regular spiking interneuron

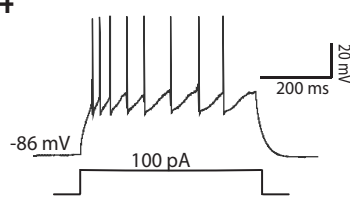

\section{5}

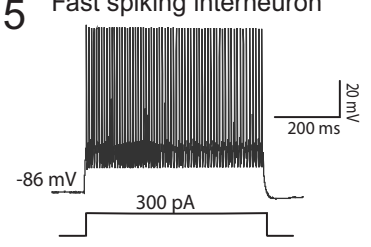

B

Channelrhodopsin

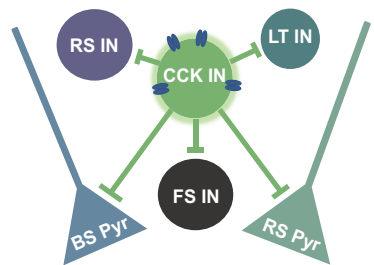

E

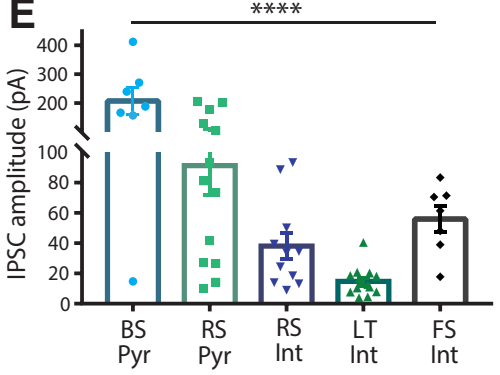

H

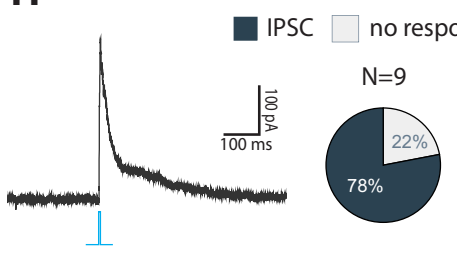

\section{I}

F
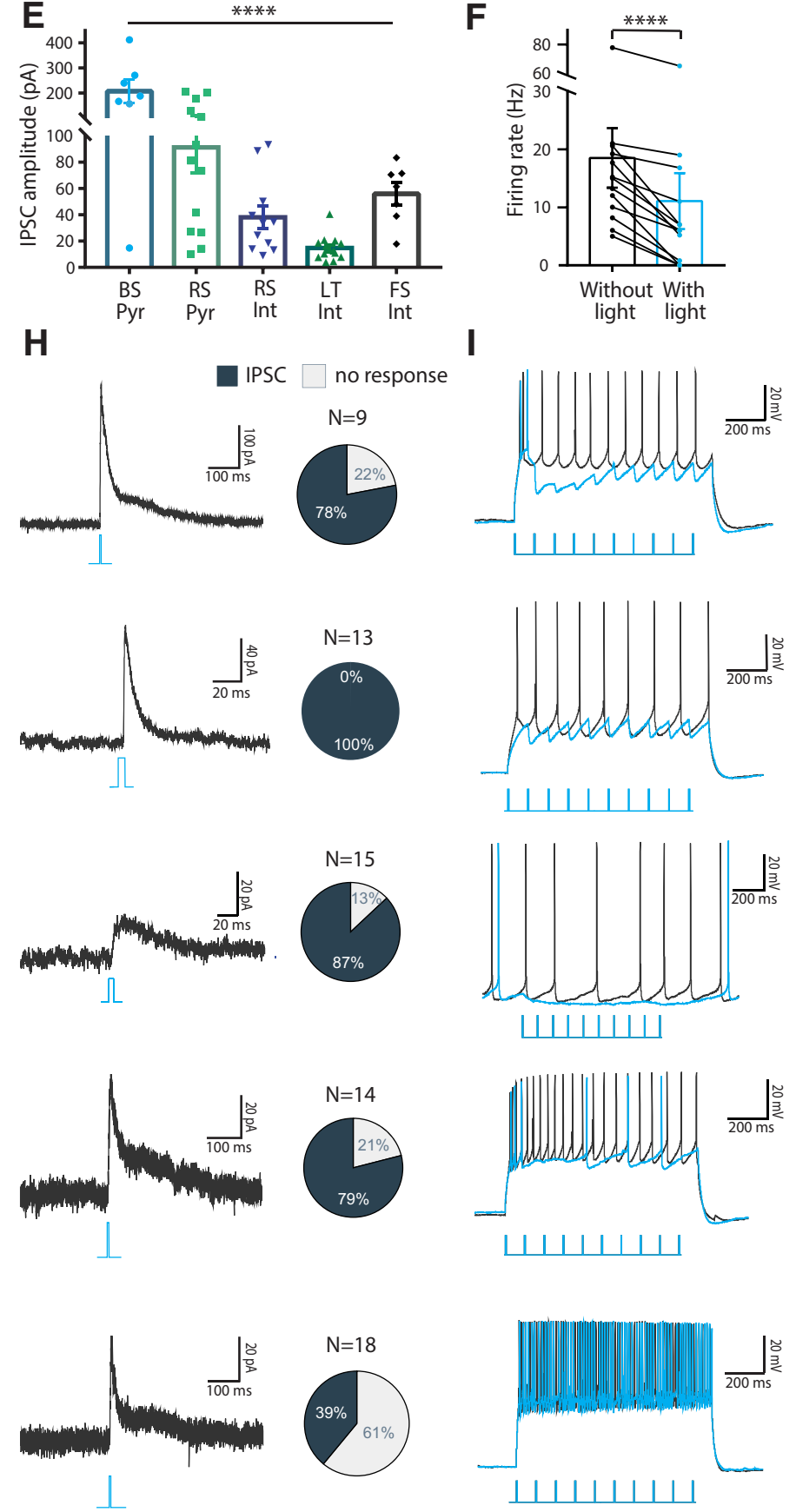

Figure 4. CCK interneurons have widespread inhibitory effects in PFC. $A$, Illustration of recording site in the mPFC. $B$, Schematic of experimental paradigm to determine the postsynaptic targets of CCK-positive interneurons by examining light-evoked postsynaptic responses in a variety of neuronal subtypes, including Burst spiking (BS) and Regular Spiking (RS) pyramidal (Pyr), Regular Spiking (RS), Low Threshold (LT) and Fast Spiking (FS) interneurons (Int). C, Histogram represents the latency of the light-evoked IPSCs that were measured in postsynaptic neurons. D, Bar graph represents the amplitude of a subset of these IPSCs before and after the application of the GABA-A receptor antagonist bicuculline (Bic), which significantly reduced the IPSC amplitudes (*** $p<$ 0.001 , paired $t$ test), confirming that the light-evoked IPS(s are predominantly mediated by GABA-A receptors. A smaller subset of interneurons also had a significant GABA-B component, which was eliminated by the GABA-B antagonist (GP52432 (CGP). Inset, Example IPSC with a significant GABA-B component, which is blocked by combined application of Bic and CGP. Calibration: 50 pA, 100 ms. $\boldsymbol{E}$, Bar graph represents amplitudes of light-evoked IPSCS measured in different neuronal types. ${ }^{* * *} p<0.0001$ (one-way ANOVA). $\boldsymbol{F}$, Bar graph shows how the (Figure legend continues.) 


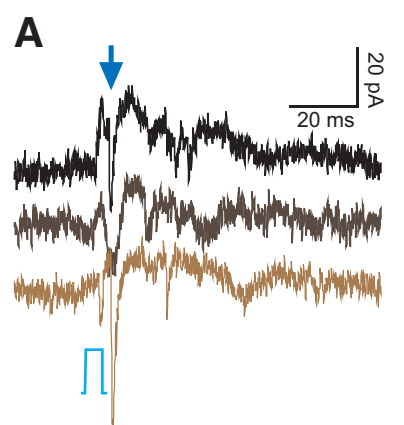

D

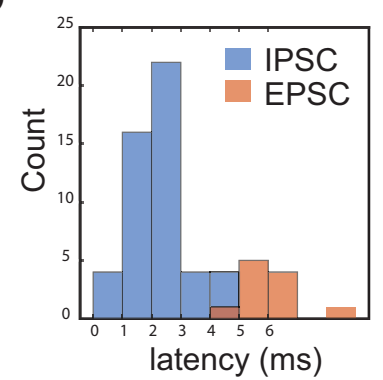

B

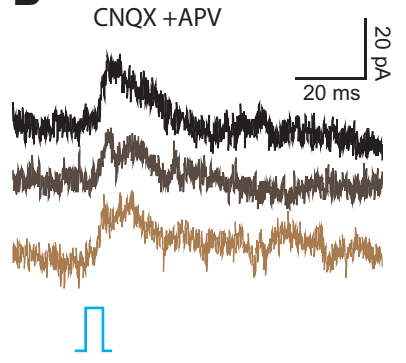

C

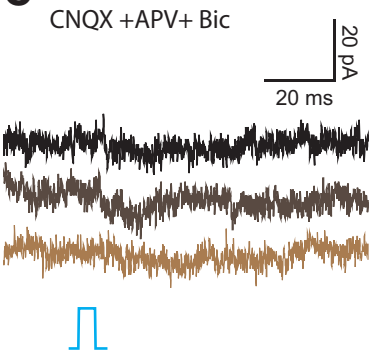

E

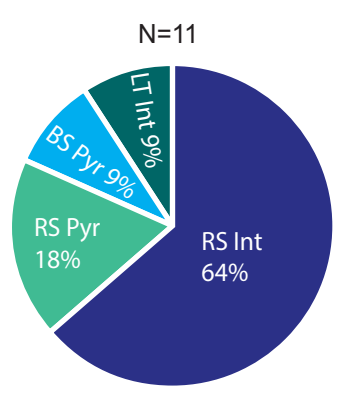

Figure 5. CCK-positive interneurons also release glutamate onto a subset of postsynaptic targets. $A$, Examples from a postsynaptic interneuron showing an EPSC (blue arrow) that consistently follows the onset of the light-evoked IPSC in successive light flashes. $\boldsymbol{B}$, Glutamate receptor blockers CNQX and APV selectively inhibit the EPSC, leaving the IPSC intact. $\boldsymbol{C}$, The further addition of a GABA-A receptor antagonist to the glutamate blockers eliminates the light-evoked postsynaptic responses completely. $\boldsymbol{D}$, Histogram of latency from light onset demonstrates the respective timing of the light-evoked EPSCS (orange) and IPSCS (blue). The EPSCS occur later than the IPSCs but within 5-9 ms of light onset. $\boldsymbol{E}$, Pie chart shows the relative proportions of cell types showing light-evoked EPSCs. Regular spiking (RS) interneurons are the most common type that show these light-evoked glutamatergic EPSCS, with examples also seen in a low threshold (LT) interneuron and in regular (RS) and burst spiking (BS) pyramidal (Pyr)neurons.

Tables 1,2), and stimulated neurotransmitter release from CCK interneurons and axons with brief pulses ( $5 \mathrm{~ms}$ ) of blue light. Light-evoked IPSCs showed rapid latency of onset from the light (Fig. $4 C$; mean latency $=2.3 \pm 0.1 \mathrm{~ms}, N=50$ ), consistent with a monosynaptic effect. The IPSCs were significantly reduced by the GABA-A receptor antagonist bicuculline (Fig. 4D; IPSC amplitude at baseline: $122 \pm 24 \mathrm{pA}$, with bicuculline: $43 \pm 12 \mathrm{pA}$; $N=13$; paired $t$ test, $\left.t_{(12)}=4.5, p<0.001\right)$. A subset of neurons also showed a prominent residual GABA-B component to the IPSC, which was reduced by subsequent addition of the GABA-B receptor antagonist CGP 52432 (IPSC amplitude in the presence of bicuculline: $94 \pm 36$ pA, IPSC amplitude after CGP $52432+$ Bic: $30 \pm 7 \mathrm{pA}, N=2$ ). The different neuronal subtypes (illustrated in Fig. 4B, G1-G5) showed distinct postsynaptic IPSC amplitudes (Fig. $4 E$; one-way ANOVA: $F_{(4,46)}=13.7, p<0.00001$ ) and connection probabilities (Fig. 4H1-H5). A high connection probability ( $\sim 80 \%-100 \%)$ was found between CCK interneurons and several neuronal subtypes, including regular and burst firing pyramidal neurons and low threshold, regular spiking interneurons. Fast spiking interneurons were the exception, with a much lower connection probability $(\sim 40 \%)$. Consistently, we found that CCK interneuron activation (by a $10 \mathrm{~Hz}$ light train for

$\leftarrow$

(Figure legend continued.) firing rate of postsynaptic neurons to a depolarizing step was inhibited by the light-evoked excitation of CCK-positive interneurons. ${ }^{* * *} p<0.0001$ (paired $t$ test). Of note, CCK interneurons could suppress spiking in all their postsynaptic targets. G1-G5, Illustrative action potential firing pattern to step current injection in different types of postsynaptic neurons recorded. $\boldsymbol{H 1 - H 5}$, Example light-evoked IPSC trace for each type of postsynaptic neuron. Inset, Pie chart represents the connection probability for each group: the proportion of neurons that show light-evoked IPSCs versus no response. I1-I5, Example traces showing the inhibition of spiking in different postsynaptic neurons by CCK interneuron stimulation. The neuronal firing elicited by current injection is shown at baseline (black) and during light activation of CCK-positive interneurons (blue). Data are mean \pm SEM.
$1 \mathrm{~s})$ significantly suppressed current-induced spiking across these postsynaptic targets $\left(N=12\right.$; paired $t$ test, $\left.t_{(11)}=7, p<0.0001\right)$ (Fig. 4I1-I5). Interestingly, in addition to the light-evoked IPSCs, a subset of neurons (11 of 79 cells) showed reliable, seemingly monosynaptic, glutamatergic EPSCs following light-evoked CCK interneuron stimulation (Fig. 5). The average EPSC amplitude across cells was $36 \pm 6 \mathrm{pA}(N=11)$ and occurred at a characteristic latency $(6.2 \pm 0.3 \mathrm{~ms}$, range: $5-9 \mathrm{~ms})$ following light onset. The light-evoked EPSCs were sensitive to the glutamate receptor antagonists CNQX and APV (EPSC amplitude before: $19.1 \pm 7.6$ pA, after CNQX +APV: no detectable EPSC, $N=3$ ), leaving the light-evoked IPSC. These excitatory responses were predominantly found in the RS interneuron subtype of prefrontal neurons, occurring in $\sim 50 \%$ of all the neurons recorded in this group. In visualizing and recording from CCK-ChR2-eGFP neurons, we did not observe any labeled neurons with either the morphological or electrophysiological features of pyramidal neurons. There is compelling evidence, however, for the expression of the glutamate transporter VGluT3 by CCK interneurons (Somogyi et al., 2004) and the ability of VGluT3 to permit glutamate corelease from GABAergic terminals (El Mestikawy et al., 2011). Thus, our observation of light-evoked glutamate release following optogenetic stimulation of CCK interneurons is consistent with the expression of the vesicular glutamate transporter VGluT3 by this class of interneurons.

Together, these data demonstrate the ability of CCK interneurons to inhibit the firing of a wide range of cell types in the $\mathrm{mPFC}$ and place CCK interneurons in a strong position to control mPFCdependent cognitive activities.

\section{Optogenetic silencing of CCK interneurons}

Next, we optogenetically silenced CCK interneurons to assess their behavioral contribution. ArchT expression was restricted to 
A

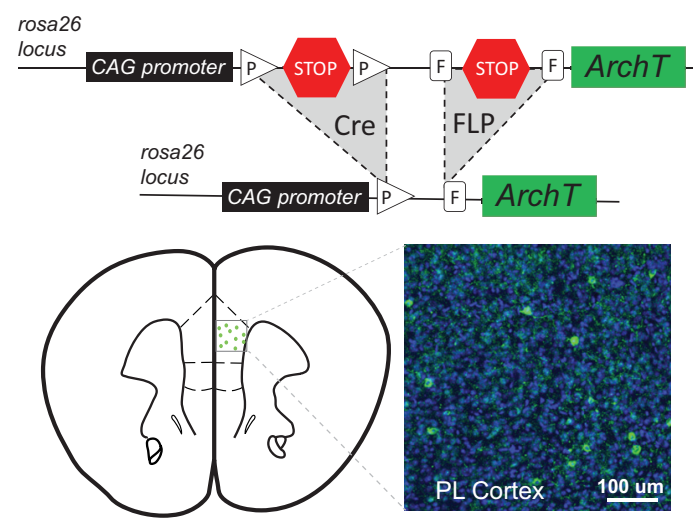

C
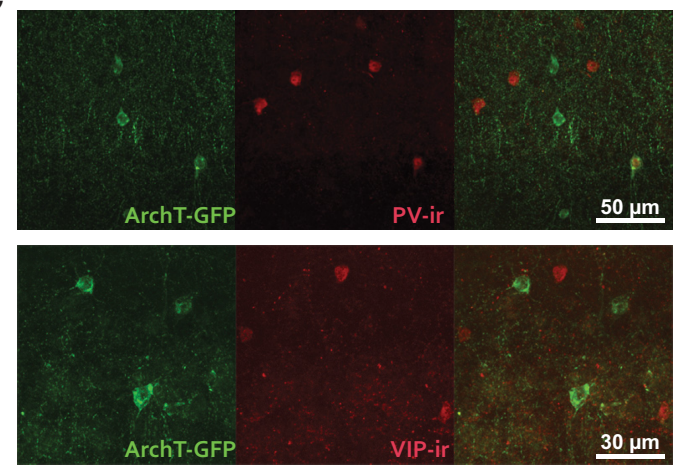

B
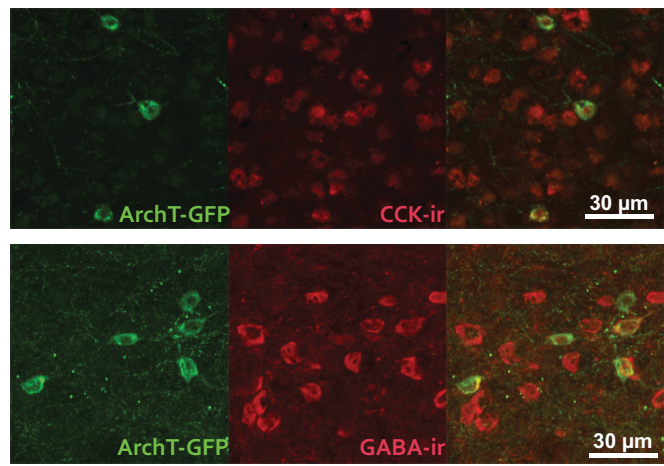

D

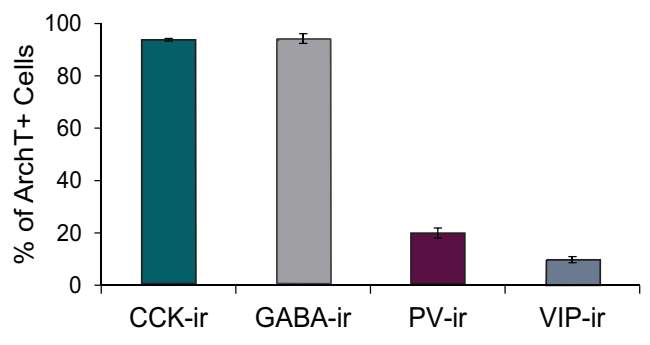

Figure 6. Intersectional genetic expression of ArchT selectively in CCK interneurons. $\boldsymbol{A}$, Top, Dual recombinase-responsive reporter allele, RC::ArchT, contains two transcriptional stop cassettes flanked by loxP and FRT sites. Bottom, Cre- and FLPe-mediated excisions result in ArchT-EGFP expression in CCK interneurons. $\boldsymbol{B}$, Representative images of immunofluorescent staining in prelimbic cortex of CCK-ArchT mice for CCK and GABA. C, Representative images of immunofluorescent staining in prelimbic cortex of CCK-ArchT mice for PV and VIP. D, Percentage of ArchT ${ }^{+}$cells in the prelimbic cortex (PL) double-labeled with GABA markers. $N=3$. Data are mean $\pm \mathrm{SEM}$.

CCK interneurons by crossing the double-transgenic CCK-Cre, Dlx5/6-FLPe mice to the RC::PFArchT-GFP line (Fig. 6A). Consistent with the pattern observed in CCK-FrePe mice, the tripletransgenic CCK-ArchT mice expressed ArchT selectively in CCK interneurons. A high proportion of ArchT-GFP + cells were immunoreactive for CCK (Fig. 6B, D; $93.8 \pm 0.9 \%$ ), and for GABA (Fig. 6B,D; $94.2 \pm 3.2 \%$ ). Furthermore, a small percentage of ArchT-GFP+ cells were immunoreactive for PV (Fig. 6C,D; $20.0 \pm 3.4 \%$ ), or VIP (Fig. $6 C, D ; 9.8 \pm 2.0 \%$ ).

In agreement with our previous findings with CCK-FrePe mice, patch-clamp recordings showed that ArchT-expressing CCK interneurons can be divided into $\mathrm{nFS}(72 \%)$ and FS (28\%) subtypes based on their firing rates (Fig. $7 A, B$ ). Notably, the amplitude of inhibitory currents evoked by a green laser pulse were greater in the FS subtype ( $154 \pm 16 \mathrm{pA}, n=5)$ compared with the nFS subtype $\left(57 \pm 7 \mathrm{pA}, n=13\right.$; unpaired $t$ test, $t_{(16)}=6.6, p<$ 0.0001 ) (Fig. 7C). The current-induced AP firing rates in both nFS ( $n=8$, two-way repeated-measures ANOVA, effect of light, $\left.F_{(1,77)}=59.3, p<0.0001\right)$ and FS subtypes $(n=4$, two-way repeated-measures ANOVA, effect of light, $F_{(1,33)}=59.8, p<$ 0.0001 ) were efficiently suppressed by light (Fig. $7 D, E)$.

\section{Behavioral function of CCK interneurons in olfactory working memory}

Working memory was tested in an olfactory DNMS task conducted in a linear three-compartment maze with scented woodchips presented in wells (Fig. 8A). On a given trial, an odor to-beremembered was presented in the sample compartment, after which the mouse traversed to the delay compartment where it was confined for $5 \mathrm{~s}$. Subsequently, in the response compartment, both matching and nonmatching odors were presented (Fig. $8 \mathrm{~A}, \mathrm{~B}$ ). A digging response to the nonmatching odor was rewarded, whereas digging in the matching odor was unrewarded and terminated the trial (Fig. 8B). For each trial, correct responses (hits and correct rejections) and incorrect responses (misses and false alarms) were scored.

We first evaluated the validity of this task in engaging working memory. Working memory is a capacity-limited process that degrades with longer retention times. Consistently, we found that performance declined with longer delay interval durations (Fig. $8 C$; one-way repeated-measures ANOVA, effect of interval, $F_{(5,20)}=10.37, p<0.0001$; linear trend, $\left.F_{(1,4)}=49.69 p<0.01\right)$, which was accounted for by the increase in false alarms with longer delay lengths (Fig. $8 D$; one-way repeated-measures ANOVA, effect of interval, $F_{(5,20)}=13.75, p<0.00001$; linear trend, $\left.F_{(1,4)}=145.71, p<0.001\right)$. Miss responses were not significantly affected by delay interval length (Fig. $8 D$; one-way repeated-measures ANOVA, effect of interval, $F_{(5,20)}=1.65, p=$ 0.192).

To silence CCK interneurons during working memory testing, triple-transgenic CCK-ArchT ${ }^{+}$and littermate control CCKArchT $^{-}$mice were implanted with optic fibers bilaterally above the prelimbic cortex. Both groups learned to perform the olfactory DNMS task with a $5 \mathrm{~s}$ delay (Fig. $9 \mathrm{~A}$; mixed ANOVA, main effect of day, $\left.F_{(11,143)}=18.65, p<0.000001\right)$, without significant differences in their acquisition (Fig. 9A; mixed ANOVA, main 
A

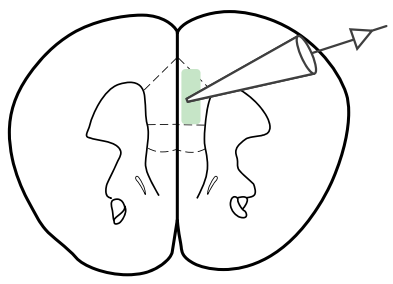

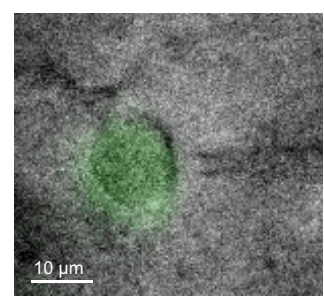

C

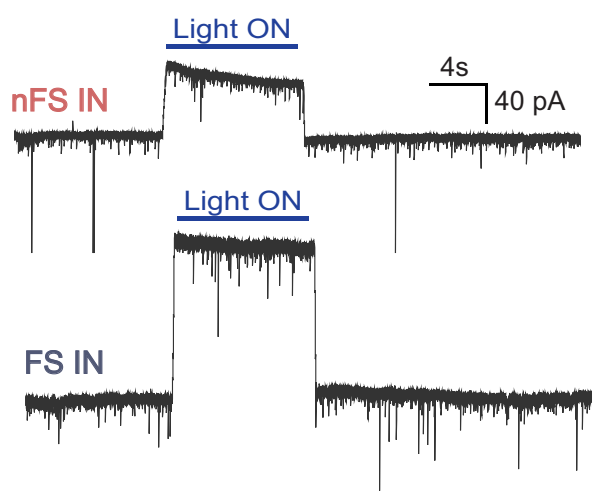

Light ON
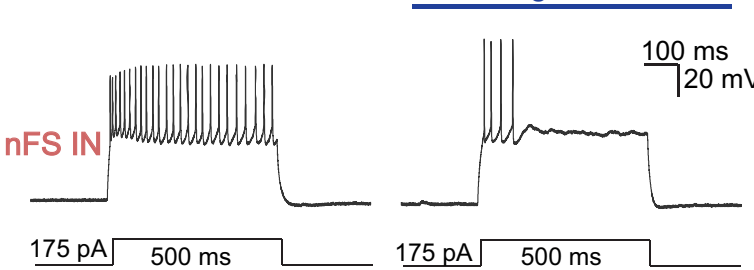

$175 \mathrm{pA} \quad 500 \mathrm{~ms}$

E

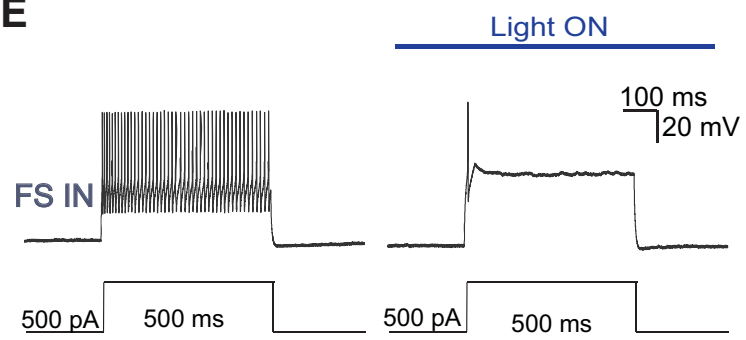

B

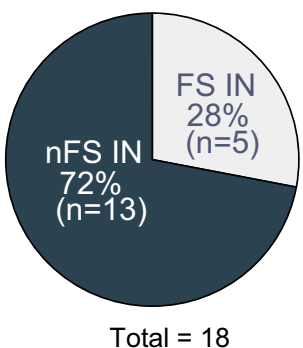

Total $=18$

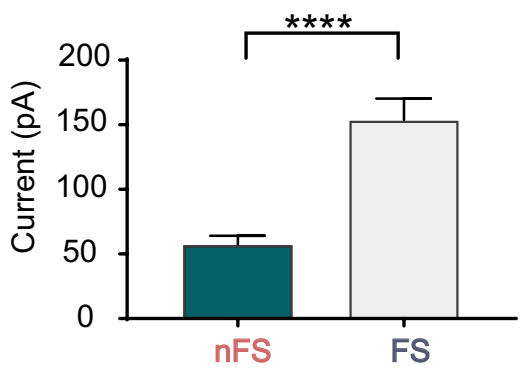

Effect of light, $P<0.0001$
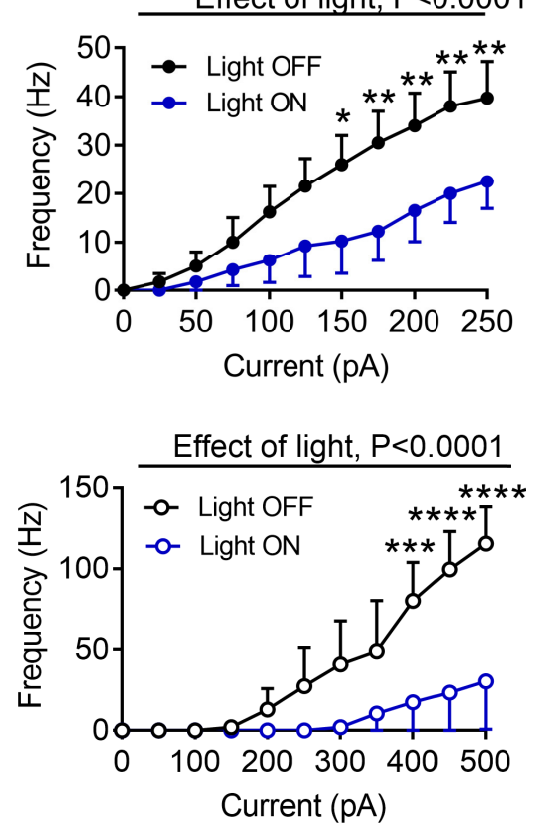

Figure 7. Electrophysiological properties of GFP-positive CCK interneurons from CCK-ArchT. A, Schematic of the mPFC with the green box representing where whole-cell patch-clamp recordings of GFP-positive CCK interneurons were performed. The IR-DIC image with GFP fluorescence overlay shows an example GFP-positive CCK interneuron. $\boldsymbol{B}$, The pie chart represents the distribution of GFP-positive CCK non-fast spiking (nFS) and fast-spiking (FS) interneurons (INs). C, A representative voltage-clamp trace at $-75 \mathrm{mV}$ shows the outward inhibitory current in response to light from an nFS IN (top) and an FS IN (bottom). Graph shows that the amplitude in response to light is greater in the FS INs compared with the nFS INs (unpaired $t$ test, $t_{(16)}=6.6$ ). ${ }^{* * * *} p<0.0001$. D, A representative current-clamp trace shows the response of an $\mathrm{nFS} I \mathrm{IN}$ to a $175 \mathrm{pA}$ depolarizing step in the absence (black) and presence (blue) of light. The input-output graph shows the firing frequency of $\mathrm{nFS} I \mathrm{Ns}(N=8)$ in response to a series of depolarizing current steps. The firing frequency is reduced in the presence of light (two-way repeated-measures ANOVA, effect of light, $\left.F_{(1,77)}=59.3, p<0.0001\right) .{ }^{*} p \leq 0.05$ (Bonferroni's post hoc test). ${ }^{* *} p \leq 0.01$ (Bonferroni's post hoc test). $\boldsymbol{E}$, A representative current-clamp trace shows the response of an $\mathrm{FS}$ IN to a $500 \mathrm{pA}$ depolarizing step in the absence (black) and presence (blue) of light. The input- output graph shows the firing frequency of FS INs $(N=4)$ in response to a series of depolarizing current steps. The firing frequency is reduced in the presence of light (two-way repeated-measures ANOVA, effect of light, $\left.F_{(1,33)}=59.8, p<0.0001\right) .{ }^{* * *} p \leq 0.001$ (Bonferroni's post hoc test). ${ }^{* * * *} p \leq 0.0001$ (Bonferroni's post hoc test). Data are mean \pm SEM.

effect of group, $\left.F_{(1,13)}=1.92, p=0.19\right)$, or criterion-level performance (Fig. $9 \mathrm{~A}$; unpaired $t$ test, $t_{(14)}=-0.54, p=0.60$ ). Previous studies have reported dissociable neural circuits underlying the sample, delay, and response phases of working memory tasks (Spellman et al., 2015; Bolkan et al., 2017). Therefore, we delivered laser light during these phases in a pseudorandomized order to evaluate the phase-specific requirement of CCK in- terneurons (Fig. 9C). Light illumination during the sample and delay phase did not significantly affect the working memory performance of CCK-ArchT ${ }^{+}$mice compared with CCK-ArchT ${ }^{-}$ controls (Fig. 9E; unpaired $t$ test, sample: $t_{(16)}=-0.02, p=0.99$; delay: $\left.t_{(16)}=0.54, p=0.60\right)$. However, CCK interneuron inhibition during the response phase significantly reduced the percentage of correct responses (Fig. 9E; mixed ANOVA, main effect 


\section{A}
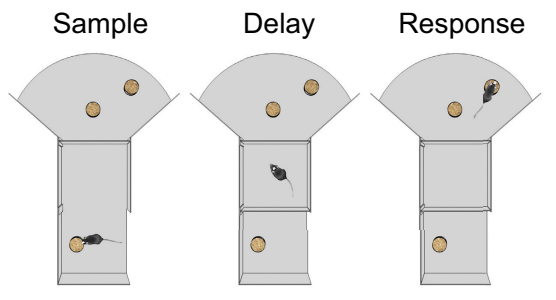

C

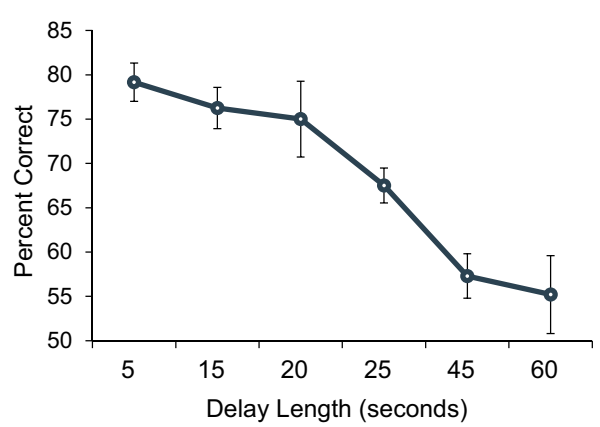

B

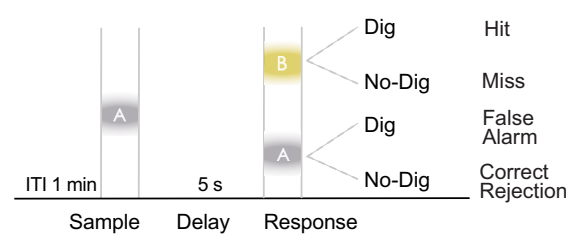

D

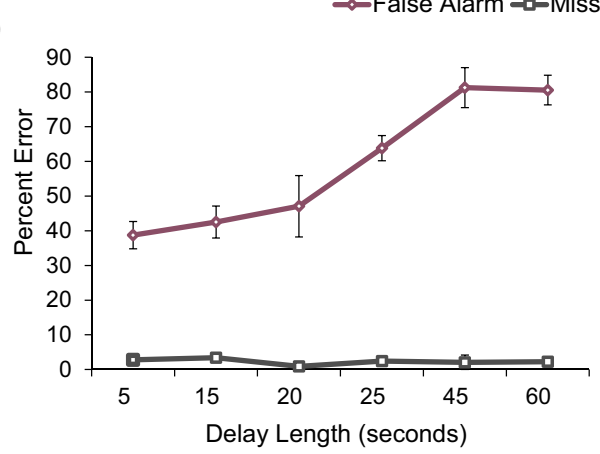

Figure 8. Olfactory DNMS performance. $\boldsymbol{A}$, Olfactory DNMS apparatus (top view) showing mouse compartment occupation during the sample, delay, and response phases. $\boldsymbol{B}$, Single-trial test paradigm and response outcomes. C, Percentage of correct responses across increasing delay lengths $(N=6)$. $\boldsymbol{D}$, Percentage of false alarms and percentage of misses across increasing delay lengths $(N=6)$. Data are mean \pm SEM.

of group, $F_{(1,16)}=10.96, p<0.01$; main effect of phase, $F_{(2,32)}=$ $15.36, p<0.0001$; phase $\times$ group interaction, $F_{(2,32)}=7.37, p<$ 0.01 ; unpaired $t$ test, $\left.t_{(16)}=6.77, p<0.00001\right)$. This impairment in performance was a result of increases in both the percentage of false alarms (Fig. 9F; mixed ANOVA, main effect of group, $F_{(1,16)}=$ $15.62, p<0.01$; main effect of phase, $F_{(2,32)}=20.96, p<0.0001$; phase $\times$ group interaction, $F_{(2,32)}=7.10, p<0.01$; unpaired $t$ test, $t_{(16)}=-5.96, p<0.0001$ ), and the percentage of misses (Fig. $9 G$; unpaired $t$ test, $\left.t_{(16)}=-2.41, p=0.029\right)$.

Our previous colocalization analysis revealed that $\sim 20 \%$ of ArchT-expressing cells in CCK-ArchT mice are positive for PV immunoreactivity (Fig. 6C,D). Thus, response phase-specific working memory impairments observed in CCK-ArchT mice could be caused in part by silencing PV interneurons. To address this, we examined the phase-specific requirement of PV interneurons in the same olfactory DMNS task. Interestingly, inhibiting PV interneurons during the task resulted in a pattern of effects distinct from CCK interneuron inhibition, and both impaired and improved performance depending on the phase (Fig. $9 H$; mixed ANOVA, phase $\times$ group interaction, $F_{(2,20)}=8.41, p<$ $0.01)$. Light delivery during the sample phase did not significantly impair working memory performance; however, a trend towards a reduction of percentage of correct responses was observed (Fig. $9 H$; unpaired $t$ test, $\left.t_{(10)}=1.89, p=0.09\right)$. Light delivery during the delay phase significantly impaired performance (Fig. $9 \mathrm{H}$; unpaired $t$ test, $\left.t_{(10)}=2.27, p<0.05\right)$, by increasing the percentage of false alarms (Fig. 9I; mixed ANOVA, phase $\times$ group interaction, $F_{(2,20)}=10.46, p<0.001$; unpaired $t$ test, $t_{(10)}=-2.68, p<$ $0.05)$, while no significant change in the percentage of misses was observed (Fig. 9J; unpaired $t$ test, $t_{(19)}=-0.05, p=0.96$ ). Light delivery during the response phase led to a greater percentage of correct responses in PV-ArchT mice compared with PV-EYFP controls (Fig. 9H; unpaired $t$ test, $t_{(10)}=-2.91, p<0.05$ ). Although no statistically significant changes were observed in a single error type, there was a trend towards a reduction in the percentage of false alarms (Fig. 9I; unpaired $t$ test, $t_{(10)}=1.95, p=0.08$ ).
Together, these results point to differential functional roles of CCK and PV interneurons during working memory. PV interneuron activity was found to be important for working memory maintenance, whereas CCK interneuron activity was required during the retrieval or use of working memory representations to guide goaldirected actions.

\section{CCK and PV interneuron inhibition does not disrupt Go/ No-go olfactory discrimination}

Changes in performance in the DNMS task may not necessarily reflect disrupted working memory and could instead result from disturbances in a number of behavioral processes, such as odor recognition and discrimination, or impulsive action. To investigate these alternatives, we performed a Go/No-go olfactory discrimination test using the same apparatus and general procedure as the DNMS test, with the exception that the reward-paired odor was kept constant on all trials throughout training and testing (Fig. 10A). Mice transitioned through the first compartment (pseudo-sample) and were confined for $5 \mathrm{~s}$ (pseudo-delay) in the middle compartment before reaching the third compartment where a response to the paired odor was rewarded (Fig. 10A). Unlike the DNMS task, no significant differences in performance were observed following phase-specific inhibition of either mPFC CCK interneurons (Fig. 10B; mixed ANOVA, main effect of group, $F<1$; main effect of phase, $F<1$; no phase $\times$ group interaction, $F_{(2,12)}=1.50, p=0.26$ ), or PV interneurons (Fig. $10 C$; mixed ANOVA, main effect of group, $F_{(1,9)}=3.27, p=0.10$; main effect of phase, $F_{(2,18)}=2.60, p=0.10$; no phase $\times$ group interaction, $\left.F_{(2,18)}=2.60, p=0.10\right)$. Therefore, the behavioral changes observed following CCK and PV interneuron inhibition in the DNMS task may be attributed to disruptions in working memory rather than to odor processing or impulsivity.

\section{Discussion}

Cortical CCK interneurons constitute a major subclass of GABAergic interneurons, which have so far escaped systematic characterization. 
A

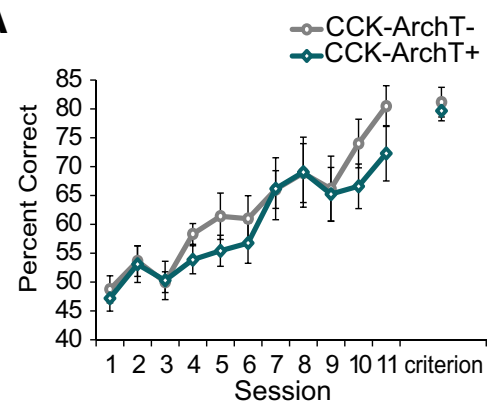

B

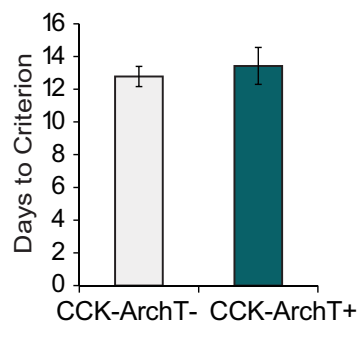

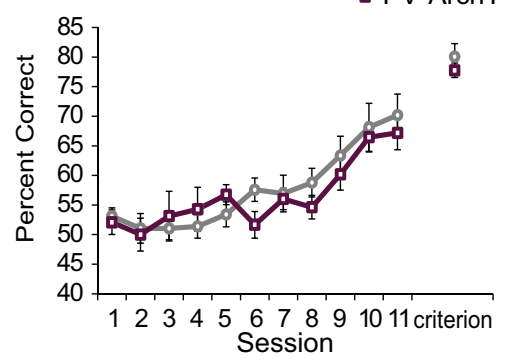

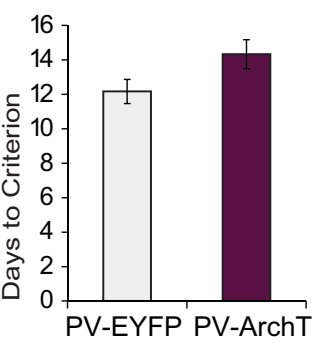

C

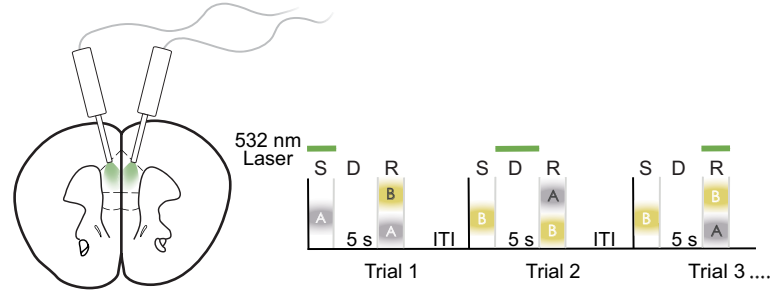

E

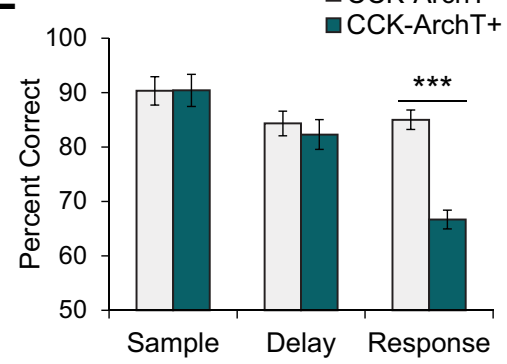

H

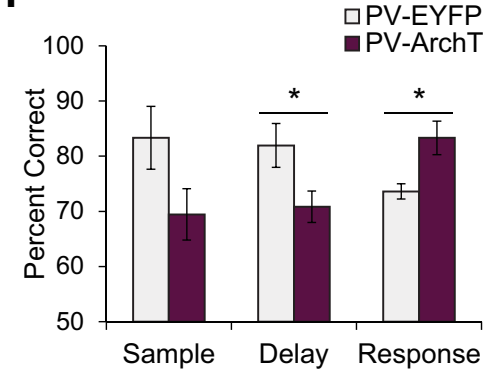

F

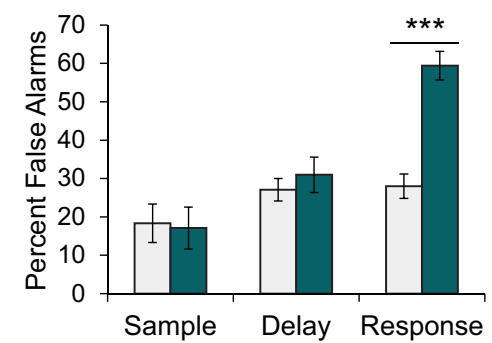

I

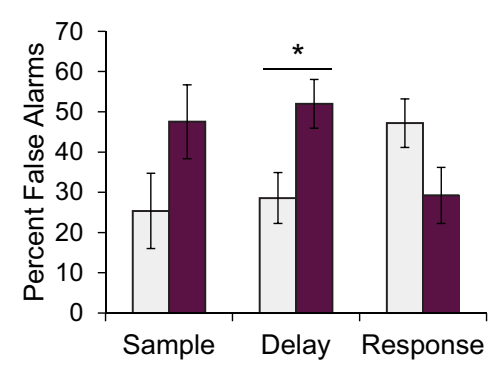

G

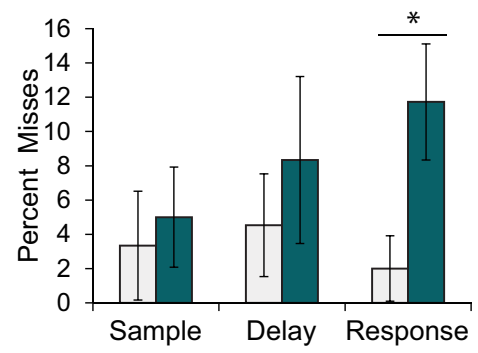

J

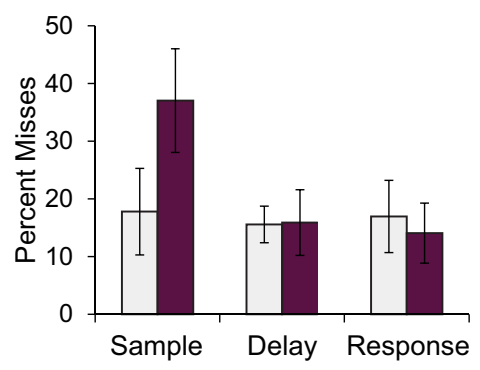

Figure 9. Phase-specific inhibition of mPFC CCK or PV interneurons during DNMS performance. $A$, Percentage of correct responses across training sessions and number of days to reach criterion performance for CCK-ArchT mice $(N=8)$ and control mice $(N=10)$. $\boldsymbol{B}$, Percentage of correct responses across training sessions and number of days to reach criterion performance for PV-ArchT mice and PV-EYFP mice ( $N=6$ each). $C$, Schematic of light illumination over the mPFC at different task phases pseudorandomized across trials with a $5 \mathrm{~s}$ delay. $\boldsymbol{D}$, Representative ArchT expression and diagram of optic fiber placement in prelimbic cortex of CCK-ArchT mice (left), and PV-ArchT mice (right). E-G, CCK-ArchT ( $N=8)$ and control ( $N=10)$ mice performance following light illumination during the sample, delay, and response phases. $\boldsymbol{H}-\boldsymbol{J}$, PV-ArchT and PV-EYFP mice ( $\boldsymbol{N}=6$ each) performance following light illumination during the sample, delay, and response phases. $\boldsymbol{E}$, $\boldsymbol{H}$, Percentage of correct responses across phases. $\boldsymbol{F}, \boldsymbol{I}$, Percentage of false alarms across phases. $\mathbf{G}, \boldsymbol{J}$, Percentage of misses across phases. ${ }^{*} p<0.05,{ }^{* * *} p<0.001$. Data are mean \pm SEM.

Using an intersectional genetic approach, we selectively targeted CCK interneurons in the $\mathrm{mPFC}$ and investigated their anatomical distribution, physiological properties, postsynaptic connectivity, and behavioral contribution in working memory.

At the anatomical level, we found that mPFC CCK interneurons spread evenly across cortical layers and comprise a large proportion of the mPFC GABA interneuron population. Differences in the laminar localization of CCK and PV interneurons, consistent with other reports (Kubota and Kawaguchi, 1997; Xu et al., 2010), point to a possible differentiation in circuit participation. Furthermore, the comparable abundance of PV and CCK interneurons in layer 5 suggests that they share inhibitory control of the major pyramidal output neurons of PFC, which project to the hippocampus and subcortical structures, such as the striatum, lateral hypothalamus, thalamus, amygdala, and spinal cord (Sesack et al., 1989; Gabbott et al., 2005; E. J. Kim et al., 2015; Zhang et al., 2016).

In converging intersectional transgenic and viral mouse models (CCK-Frepe, CCK-ArchT, and CCK-ChR2), our electrophysiological characterization of CCK interneurons revealed an unusual electrophysiological heterogeneity. The majority of CCK neurons exhibited spiking features that would lead to their classification as non-Fast spiking interneurons with intrinsic properties that would predict their rapid recruitment by incoming stimuli. A substantial minority of CCK interneurons, by contrast, exhibited Fast-spiking characteristics and a right-shifted input- 
A

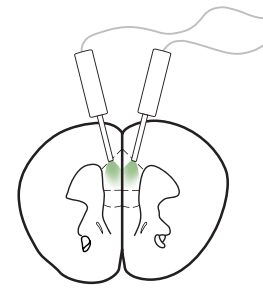

B

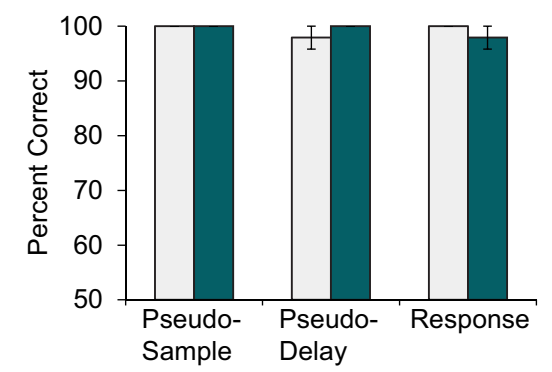

$532 \mathrm{~nm}$ Laser

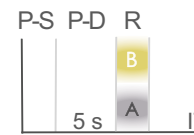

Trial 1

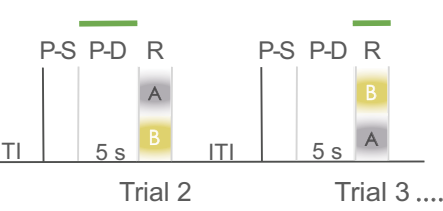

Trial 2

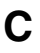

口PV-EYFP QPV-ArchT

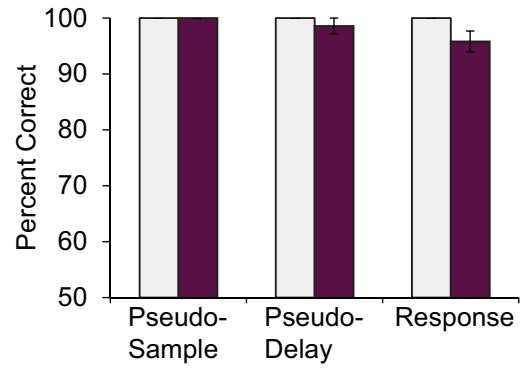

Figure 10. Phase-specific inhibition of mPFC CCK or PV interneurons during Go/No-go odor discrimination performance. $A$, Schematic of light illumination at different task phases across trials. $B, C$, Percentage of correct responses following light illumination during the pseudo-sample, pseudo-delay, and response phases of (B) CCK-ArchT and control ( $N=4$ each), and (C) PV-ArchT $(N=6)$ and PV-EYFP $(N=5)$. Data are mean \pm SEM.

output curve. This minority is consistent with the subgroup of intersectionally labeled CCK interneurons in both CCK-FrePe and CCK-ArchT mice with immunostaining for PV, widely considered a marker of FS activity (Kubota and Kawaguchi, 1997; Galarreta and Hestrin, 1999; Gibson et al., 1999; Hu et al., 2017). The proportions of the PV-negative and PV-positive CCK interneurons from our anatomical analysis are consistent with the proportions of nFS CCK interneurons and FS CCK interneurons that we identified in the slice electrophysiology experiments.

To identify the targets of prefrontal CCK interneurons, we optogenetically stimulated CCK interneurons labeled intersectionally with ChR2 while recording from potential postsynaptic neurons. These recordings from multiple subtypes of prefrontal neurons reveal that CCK interneurons possess a postsynaptic profile distinct from that of any previously characterized interneuron group. They target a striking variety of different subtypes of pyramidal neurons and GABAergic interneurons with high connection probability. The intrinsic and spiking properties of the pyramidal neurons targeted are consistent with Regular-spiking intratelencephalic corticostriatal neurons as well as Burst spiking corticothalamic neurons (Hattox and Nelson, 2007; Llano and Sherman, 2009). The interneurons targeted include the lowthreshold spiking and Regular spiking subgroups, broadly consistent with the somatostatin (SST) and VIP subclasses (Ma et al., 2006; Prönneke et al., 2015; Batista-Brito et al., 2017; Nigro et al., 2018). Of the different cellular targets examined, only the Fast spiking, likely PV-positive, interneurons showed a lower probability of being synaptically targeted by the CCK neuronal population. Overall, the diverse pattern of CCK interneuron innervation suggests that this population is positioned to exert a sudden and strong inhibition of prefrontal cortical activity.

The connectivity pattern of CCK interneurons is distinct from that of other interneuron types in the cortex, such as VIP, SST, and PV interneurons. VIP interneurons preferentially innervate PV and SST interneurons forming a disinhibitory circuit (Lee et al., 2013; Pi et al., 2013; Kepecs and Fishell, 2014). SST interneurons target pyramidal neuron dendrites and PV and VIP in-

terneurons, and avoid innervating other SST interneurons (Xu et al., 2013; Tremblay et al., 2016), whereas PV interneurons target other PV interneurons and pyramidal cells (Pfeffer et al., 2013; Jiang et al., 2015). Thus, the broad innervation profile of the CCK interneurons in connecting to output pyramidal cells as well as all the intermediary interneuron types in the cortex is distinct from the connectivity profile of any one cortical interneuron group and potentially places them in a distinctive regulatory role.

It is well known that widespread inhibition is a mechanism that can synchronize neural activity, which can be important for information processing by prioritizing task relevant information over background noise (Dipoppa et al., 2016). This would support the role for CCK interneurons during the response phase of a working memory task in which synchronized prefrontal activity is a feature (Goldman-Rakic, 1995; Compte et al., 2000). Of note, our work measures the many to one connection probability from CCK interneurons to a given postsynaptic neuron type. However, more information about the local inhibitory circuits controlled by CCK interneurons might be gleaned by measuring the one to one connection probabilities between pairs of neurons. In addition, the inputs to CCK interneurons and which of the postsynaptic connections made by CCK interneurons are involved in different phases of the working memory task are yet to be characterized and will shed more light on the cortical circuits involved in working memory.

Coordination within precise neural circuits underlies discrete cognitive epochs of working memory, namely, the processing, storage, and use of information to guide goal-directed behaviors (Baddeley, 2003). These epochs correspond to the sample, delay, and response phases of delayed-nonmatch-to-sample tests of working memory (Dudchenko, 2004), and involve activity in the mPFC (Jones and Wilson, 2005; Hyman et al., 2010; Parnaudeau et al., 2013; Ito et al., 2015; Spellman et al., 2015; Bolkan et al., 2017). Optogenetic silencing of CCK or PV interneurons during specific phases of the working memory task resulted in different behavioral changes. We found that PV interneuron activity was important for working memory maintenance, while CCK interneuron activity was required during the retrieval or use of working memory representations to guide goal-directed actions.

Approximately $20 \%$ of intersectionally labeled CCK interneurons in both CCK-FrePe and CCK-ArchT mice expressed PV, while the remaining $80 \%$ were PV-negative. It is well established that nearly all PV-positive interneurons display FS activity (Kawaguchi and Kubota, 1997; Galarreta and Hestrin, 1999; Gibson et al., 1999; Hu et al., 2017). Therefore, the PV-positive and PV-negative CCK interneurons account for FS CCK interneurons and $\mathrm{nFS} \mathrm{CCK}$ interneurons, respectively. It is likely that these PV-expressing CCK interneurons were labeled with ArchT in PV-ArchT mice as a subgroup of the PV interneuron population. Importantly, however, inhibiting the PV-expressing CCK interneurons during the response phase did not impair working memory performance. Therefore, our findings suggest that working memory retrieval may require the activity of nFS CCK interneurons, 
but not FS CCK or PV interneurons. Future experiments will have to address how the FS CCK interneurons functionally differ from other PV interneurons that do not express CCK.

During the delay interval, mPFC neurons display temporally limited sequential firing. It has been proposed that this $\mathrm{mPFC}$ delay activity directly represents sensory features of information maintained in working memory (Goldman-Rakic, 1995; Fujisawa et al., 2008; Liu et al., 2014; Bolkan et al., 2017), or alternatively, that it helps keep active the sensory representations that are encoded in primary sensory cortex (Postle et al., 2003). This $\mathrm{mPFC}$ delay activity is facilitated by inputs arriving from the mediodorsal thalamus (Parnaudeau et al., 2013; Bolkan et al., 2017; Schmitt et al., 2017), which appear to preferentially excite PV interneurons (Kuroda et al., 2004; Rotaru et al., 2005; Delevich et al., 2015; Schmitt et al., 2017), contributing to suppression of weakly active pyramidal neurons and sparse delay activity (Schmitt et al., 2017). PV interneuron activity may be sustained through recurrent excitation of pyramidal neurons in layers II/III allowing for reverberation of activity within the mPFC (GoldmanRakic, 1996; Moghaddam and Adams, 1998; Constantinidis and Wang, 2004; Wang et al., 2013). Persistent activity during cognitive performance is associated with gamma oscillations (Fries, 2009; Buzsáki and Wang, 2012; Cho et al., 2015; H. Kim et al., 2016; Lundqvist et al., 2016). Gamma band power and the frequency of gamma bursts increase with working memory load, both as a function of the number of items-to-be-remembered and retention interval length (Howard et al., 2003; Roux et al., 2012; Honkanen et al., 2015; Kornblith et al., 2016; Lundqvist et al., 2016). Importantly, gamma oscillations in the $\mathrm{mPFC}$ require $\mathrm{PV}$ interneurons for the fast synchronization of pyramidal cells (Freund, 2003; Tukker et al., 2007; Cardin et al., 2009; Sohal et al., 2009). This has been demonstrated bidirectionally as selective optogenetic activation of PV interneurons in vivo at $40 \mathrm{~Hz}$ enhances gamma frequency power (Cardin et al., 2009; Cho et al., 2015), while PV interneuron inhibition suppresses these oscillations (Sohal et al., 2009). Therefore, the working memory impairment we observed upon inhibiting PV interneurons during the delay period may involve diminished gamma frequency oscillations and disrupted persistent delay activity.

The retrieval and adaptive use of information during the response phase of working memory requires coordination between the $\mathrm{mPFC}$ and long-range memory-associated networks. In particular, coupling of mPFC and dorsal hippocampus (dHPC) activity has been consistently implicated in successful choice behavior during spatial working memory performance (Kupferschmidt and Gordon, 2018). Both local theta and gamma oscillations and single units in the mPFC become synchronized with dHPC theta oscillations at the choice point of delayednonmatch-to-sample tasks, particularly on correct trials when working memory is appropriately used (Fujisawa and Buzsáki, 2011; O’Neill et al., 2013; Hallock et al., 2016; Tamura et al., 2017). Furthermore, theta frequency synchronization between dHPC and mPFC has been observed during goal selection in long-term memory tasks (Benchenane et al., 2010; Preston and Eichenbaum, 2013; Place et al., 2016), and may therefore generally reflect the retrieval of task-relevant representations to guide decision-making. In the dHPC, CCK interneurons have been implicated in maintaining local theta power as well as precise and stable spatial representations (Del Pino et al., 2017). Thus, inhibiting $\mathrm{mPFC}$ CCK interneurons during the response phase may disrupt the retrieval of task-relevant representations and theta frequency synchronization between the MPFC and dHPC, leading to impaired working memory performance.
In addition to working memory, the mPFC has been implicated in long-term memory retrieval, suppressing impulsive actions, and selecting task-appropriate behaviors (Euston et al., 2012; H. Kim et al., 2016). These processes are unlikely to be affected by either CCK or PV inhibition since performance on the Go/No-go odor discrimination task was left intact. This experiment served as an important control because it required mice to perform the same actions without engaging working memory. The deficits we observed with inhibition during the delay interval and response period may be accounted for by disruptions to precise processes involved in each phase of working memory performance. PV interneuron inhibition during the delay interval may have affected the maintenance of content information, attention, or resistance to distractors (Lara and Wallis, 2015; Riley and Constantinidis, 2016); while CCK interneuron response inhibition may have impaired task-dependent retrieval, the representation of rules or their use to organize goal-directed behaviors (Euston et al., 2012; Preston and Eichenbaum, 2013).

Of note, CCK-GABA cells are the only type of cortical interneuron that express CB1 receptors (Marsicano and Lutz, 1999; Takács et al., 2015). Consistent with our finding, impaired working memory is a well-established consequence of CB1 receptor binding by exogenous cannabinoids applied systemically and locally in the mPFC (Varvel et al., 2001; Varvel and Lichtman, 2002; De Melo et al., 2005; Avdesh et al., 2013). Adolescent cannabinoid use has been implicated in the increased risk of developing schizophrenia (Moore et al., 2007), a condition in which working memory impairments are a core deficit (Forbes et al., 2009; Gold et al., 2010; Anticevic et al., 2011). In addition, schizophrenia postmortem studies found decreased levels of CB1 receptors at both the mRNA and protein level in the PFC (Eggan et al., 2008, 2010). In the same area of the same subjects, mRNA levels of CCK were also found to be significantly reduced (Eggan et al., 2008; Curley and Lewis, 2012). While both CCK and PV interneurons provide strong feedforward inhibition onto pyramidal neurons, differences in their electrophysiological properties, synaptic receptors, neurotransmitter release, and postsynaptic connectivity can collectively shape their unique roles in cortical processing and cognition (Daw et al., 2009; Armstrong and Soltesz, 2012). Our results support the idea that CCK and PV interneurons in the $\mathrm{mPFC}$ differentially regulate specific processes involved in working memory. PV interneurons may support information maintenance while CCK interneurons contribute to task-appropriate behavioral responding. Dysfunction in CCK interneurons in the mPFC may underlie a distinct aspect of the working memory impairment observed in cannabinoid use as well as neuropsychiatric illnesses, such as schizophrenia.

CCK interneurons are a large and complex class of GABA interneurons whose functional role we are only beginning to elucidate. Here, we have used an intersectional approach to systematically characterize, for the first time, their anatomical distribution, physiological properties, postsynaptic connectivity, and behavioral contribution in working memory. We find that there are two major subclasses of CCK interneurons, and their differing characteristics will shape their recruitment. CCK interneurons as a population target a variety of different subtypes of pyramidal neurons and other GABAergic interneurons, implying that they can exert strong and certain inhibition of mPFC activity. Acute optogenetic inhibition of this population impairs working memory retrieval in a phase-specific manner, suggesting that the coordinated activity of CCK interneurons may act as a trigger to reset ongoing network activity and initiate goal-directed actions. 


\section{References}

Abbas AI, Sundiang MJ, Henoch B, Morton MP, Bolkan SS, Park AJ, Harris AZ, Kellendonk C, Gordon JA (2018) Somatostatin interneurons facilitate hippocampal-prefrontal synchrony and prefrontal spatial encoding. Neuron 100:926-939.e3.

Anticevic A, Repovs G, Corlett PR, Barch DM (2011) Negative and nonemotional interference with visual working memory in schizophrenia. Biol Psychiatry 70:1159-1168.

Armstrong C, Soltesz I (2012) Basket cell dichotomy in microcircuit function. J Physiol 590:683-694.

Avdesh A, Hoe Y, Martins RN, Martin-Iverson MT (2013) Pharmacological effects of cannabinoids on the reference and working memory functions in mice. Psychopharmacology (Berl) 225:483-494.

Awatramani R, Soriano P, Rodriguez C, Mai JJ, Dymecki SM (2003) Cryptic boundaries in roof plate and choroid plexus identified by intersectional gene activation. Nat Genet 35:70-75.

Baddeley A (2003) Working memory: looking back and looking forward. Nat Rev Neurosci 4:829-839.

Bang SJ, Jensen P, Dymecki SM, Commons KG (2012) Projections and interconnections of genetically defined serotonin neurons in mice. Eur J Neurosci 35:85-96.

Bartos M, Elgueta C (2012) Functional characteristics of parvalbumin- and cholecystokinin-expressing basket cells. J Physiol 590:669-681.

Basu J, Srinivas KV, Cheung SK, Taniguchi H, Huang ZJ, Siegelbaum SA (2013) A cortico-hippocampal learning rule shapes inhibitory microcircuit activity to enhance hippocampal information flow. Neuron 79:12081221.

Basu J, Zaremba JD, Cheung SK, Hitti FL, Zemelman BV, Losonczy A, Siegelbaum SA (2016) Gating of hippocampal activity, plasticity, and memory by entorhinal cortex long-range inhibition. Science 351:aaa5694.

Batista-Brito R, Vinck M, Ferguson KA, Chang JT, Laubender D, Lur G, Mossner JM, Hernandez VG, Ramakrishnan C, Deisseroth K, Higley MJ, Cardin JA (2017) Developmental dysfunction of VIP interneurons impairs cortical circuits. Neuron 95:884-895.e9.

Baude A, Bleasdale C, Dalezios Y, Somogyi P, Klausberger T (2007) Immunoreactivity for the GABAA receptor $\alpha 1$ subunit, somatostatin and connexin36 distinguishes axoaxonic, basket, and bistratified interneurons of the rat hippocampus. Cereb Cortex 17:2094-2107.

Benchenane K, Peyrache A, Khamassi M, Tierney PL, Gioanni Y, Battaglia FP, Wiener SI (2010) Coherent theta oscillations and reorganization of spike timing in the hippocampal- prefrontal network upon learning. Neuron 66: 921-936.

Bezaire MJ, Soltesz I (2013) Quantitative assessment of CA1 local circuits: knowledge base for interneuron-pyramidal cell connectivity. Hippocampus 23:751-785.

Bodor AL, Katona I, Nyíri G, Mackie K, Ledent C, Hájos N, Freund TF (2005) Endocannabinoid signaling in rat somatosensory cortex: laminar differences and involvement of specific interneuron types. J Neurosci 25:6845-6856.

Bolkan SS, Stujenske JM, Parnaudeau S, Spellman TJ, Rauffenbart C, Abbas AI, Harris AZ, Gordon JA, Kellendonk C (2017) Thalamic projections sustain prefrontal activity during working memory maintenance. Nat Neurosci 20:987-996.

Buzsáki G, Wang XJ (2012) Mechanisms of gamma oscillations. Annu Rev Neurosci 35:203-225.

Canetta S, Bolkan S, Padilla-Coreano N, Song LJ, Sahn R, Harrison NL, Gordon JA, Brown A, Kellendonk C (2016) Maternal immune activation leads to selective functional deficits in offspring parvalbumin interneurons. Mol Psychiatry 21:956-968.

Cardin JA, Carlén M, Meletis K, Knoblich U, Zhang F, Deisseroth K, Tsai LH, Moore CI (2009) Driving fast-spiking cells induces gamma rhythm and controls sensory responses. Nature 459:663-667.

Carter E, Wang XJ (2007) Cannabinoid-mediated disinhibition and working memory: dynamical interplay of multiple feedback mechanisms in a continuous attractor model of prefrontal cortex. Cereb Cortex 17 [Suppl 1]:16-26.

Cea-del Rio CA, McBain CJ, Pelkey KA (2012) An update on cholinergic regulation of cholecystokinin-expressing basket cells. J Physiol 590:695-702.

Cho KK, Hoch R, Lee AT, Patel T, Rubenstein JL, Sohal VS (2015) Gamma rhythms link prefrontal interneuron dysfunction with cognitive inflexibility in dlx5/6 ${ }^{+/-}$mice. Neuron 85:1332-1343.

Compte A, Brunel N, Goldman-Rakic PS, Wang XJ (2000) Synaptic mech- anisms and network dynamics underlying spatial working memory in a cortical network model. Cereb Cortex 10:910-923.

Constantinidis C, Wang XJ (2004) A neural circuit basis for spatial working memory. Neuroscientist 10:553-565.

Cope DW, Maccaferri G, Márton LF, Roberts JD, Cobden PM, Somogyi P (2002) Cholecystokinin-immunopositive basket and Schaffer collateralassociated interneurones target different domains of pyramidal cells in the CA1 area of the rat hippocampus. Neuroscience 109:63-80.

Curley AA, Lewis DA (2012) Cortical basket cell dysfunction in schizophrenia. J Physiol 590:715-724.

Curtis CE, D'Esposito M (2004) The effects of prefrontal lesions on working memory performance and theory. Cogn Affect Behav Neurosci 4:528539.

Daw MI, Tricoire L, Erdelyi F, Szabo G, McBain CJ (2009) Asynchronous transmitter release from cholecystokinin-containing inhibitory interneurons is widespread and target-cell independent. J Neurosci 29:11112-11122.

Del Pino I, Brotons-Mas JR, Marques-Smith A, Marighetto A, Frick A, Marín O, Rico B (2017) Abnormal wiring of CCK ${ }^{+}$basket cells disrupts spatial information coding. Nat Neurosci 20:784-792.

Delevich K, Tucciarone J, Huang ZJ, Li B (2015) The mediodorsal thalamus drives feedforward inhibition in the anterior cingulate cortex via parvalbumin interneurons. J Neurosci 35:5743-5753.

De Melo LC, Cruz AP, Valentim SJ, Marinho AR, Mendonça JB, NakamuraPalacios EM (2005) $\Delta$ 9-THC administered into the medial prefrontal cortex disrupts the spatial working memory. Psychopharmacology (Berl) 183:54-64.

Dimidschstein J, Chen Q, Tremblay R, Rogers SL, Saldi GA, Guo L, Xu Q, Liu R, Lu C, Chu J, Grimley JS, Krostag AR, Kaykas A, Avery MC, Rashid MS, Baek M, Jacob AL, Smith GB, Wilson DE, Kosche G, et al. (2016) A viral strategy for targeting and manipulating interneurons across vertebrate species. Nat Neurosci 19:1743-1749.

Dipoppa M, Szwed M, Gutkin BS (2016) Controlling working memory operations by selective gating: the roles of oscillations and synchrony. Adv Cogn Psychol 12:209-232.

Douglas RJ, Martin KA (2004) Neuronal circuits of the neocortex. Annu Rev Neurosci 27:419-451.

Dudchenko PA (2004) An overview of the tasks used to test working memory in rodents. Neurosci Biobehav Rev 28:699-709.

Dudok B, Barna L, Ledri M, Szabó SI, Szabadits E, Pintér B, Woodhams SG, Henstridge CM, Balla GY, Nyilas R, Varga C, Lee SH, Matolcsi M, Cervenak J, Kacskovics I, Watanabe M, Sagheddu C, Melis M, Pistis M, Soltesz I, et al. (2015) Cell-specific STORM super-resolution imaging reveals nanoscale organization of cannabinoid signaling. Nat Neurosci 18:75-86.

Eggan SM, Hashimoto T, Lewis DA (2008) Reduced cortical cannabinoid 1 receptor messenger RNA and protein expression in schizophrenia. Arch Gen Psychiatry 65:772-784.

Eggan SM, Stoyak SR, Verrico CD, Lewis DA (2010) Cannabinoid CB1 receptor immunoreactivity in the prefrontal cortex: comparison of schizophrenia and major depressive disorder. Neuropsychopharmacology 35: 2060-2071.

El Mestikawy S, Wallén-Mackenzie A, Fortin GM, Descarries L, Trudeau LE (2011) From glutamate co-release to vesicular synergy: vesicular glutamate transporters. Nat Rev Neurosci 12:204-216.

Engleka KA, Manderfield LJ, Brust RD, Li L, Cohen A, Dymecki SM, Epstein JA (2012) Islet1 derivatives in the heart are of both neural crest and second heart field origin. Circ Res 110:922-926.

Eriksson J, Vogel EK, Lansner A, Bergström F, Nyberg L (2015) Neurocognitive architecture of working memory. Neuron 88:33-46.

Euston DR, Gruber AJ, McNaughton BL (2012) The role of medial prefrontal cortex in memory and decision making. Neuron 76:1057-1070.

Forbes NF, Carrick LA, McIntosh AM, Lawrie SM (2009) Working memory in schizophrenia: a meta-analysis. Psychol Med 39:889-905.

Freund TF (2003) Interneuron diversity series: rhythm and mood in perisomatic inhibition. Trends Neurosci 26:489-495.

Freund TF, Katona I (2007) Perisomatic inhibition. Neuron 56:33-42.

Fries P (2009) Neuronal gamma-band synchronization as a fundamental process in cortical computation. Annu Rev Neurosci 32:209-224.

Fujisawa S, Buzsáki G (2011) A $4 \mathrm{~Hz}$ oscillation adaptively synchronizes prefrontal, VTA, and hippocampal activities. Neuron 72:153-165.

Fujisawa S, Amarasingham A, Harrison MT, Buzsáki G (2008) Behaviordependent short-term assembly dynamics in the medial prefrontal cortex. Nat Neurosci 11:823-833. 
Fung SJ, Fillman SG, Webster MJ, Shannon Weickert C (2014) Schizophrenia and bipolar disorder show both common and distinct changes in cortical interneuron markers. Schizophr Res 155:26-30.

Fuster JM, Alexander GE (1971) Neuron activity related to short-term memory. Science 173:652-654.

Gabbott PL, Warner TA, Jays PR, Salway P, Busby SJ (2005) Prefrontal cortex in the rat: projections to subcortical autonomic, motor, and limbic centers. J Comp Neurol 492:145-177.

Galarreta M, Hestrin S (1999) A network of fast-spiking cells in the neocortex connected by electrical synapses. Nature 402:72-75.

Gibson JR, Beierlein M, Connors BW (1999) Two networks of electrically coupled inhibitory neurons in neocortex. Nature 402:75-79.

Gold JM, Hahn B, Zhang WW, Robinson BM, Kappenman ES, Beck VM, Luck SJ (2010) Reduced capacity but spared precision and maintenance of working memory representations in schizophrenia. Arch Gen Psychiatry 67:570-577.

Goldman-Rakic PS (1995) Cellular basis of working memory. Neuron 14: 477-485.

Goldman-Rakic PS (1996) Regional and cellular fractionation of working memory. Proc Natl Acad Sci U S A 93:13473-13480.

Halasy K, Buhl EH, Lörinczi Z, Tamás G, Somogyi P (1996) Synaptic target selectivity and input of GABAergic basket and bistratified interneurons in the CA1 area of the rat hippocampus. Hippocampus 6:306-329.

Hallock HL, Wang A, Griffin AL (2016) Ventral midline thalamus is critical for hippocampal: prefrontal synchrony and spatial working memory. J Neurosci 36:8372-8389.

Harris KD, Hochgerner H, Skene NG, Magno L, Katona L, Bengtsson Gonzales C, Somogyi P, Kessaris N, Linnarsson S, Hjerling-Leffler J (2018) Classes and continua of hippocampal CA1 inhibitory neurons revealed by single-cell transcriptomics. PLoS Biol 16:e2006387.

Hashimoto T, Arion D, Unger T, Maldonado-Avilés JG, Morris HM, Volk DW, Mirnics K, Lewis DA (2008) Alterations in GABA-related transcriptome in the dorsolateral prefrontal cortex of subjects with schizophrenia. Mol Psychiatry 13:147-161.

Hattox AM, Nelson SB (2007) Layer V neurons in mouse cortex projecting to different targets have distinct physiological properties. J Neurophysiol 98:3330-3340.

Hefft S, Jonas P (2005) Asynchronous GABA release generates long-lasting inhibition at a hippocampal interneuron-principal neuron synapse. Nat Neurosci 8:1319-1328.

Honkanen R, Rouhinen S, Wang SH, Palva JM, Palva S (2015) Gamma oscillations underlie the maintenance of feature-specific information and the contents of visual working memory. Cereb Cortex 25:3788-3801.

Howard MW, Rizzuto DS, Caplan JB, Madsen JR, Lisman J, AschenbrennerScheibe R, Schulze-Bonhage A, Kahana MJ (2003) Gamma oscillations correlate with working memory load in humans. Cereb Cortex 13:13691374.

Hu H, Gan J, Jonas P (2014) Fast-spiking, parvalbumin ${ }^{+}$GABAergic interneurons: from cellular design to microcircuit function. Science 345: 1255263.

Hu JS, Vogt D, Sandberg M, Rubenstein JL (2017) Cortical interneuron development: a tale of time and space. Development 144:3867-3878.

Hyman JM, Zilli EA, Paley AM, Hasselmo ME (2010) Working memory performance correlates with prefrontal-hippocampal theta interactions but not with prefrontal neuron firing rates. Front Integr Neurosci 4:2.

Isaacson JS, Scanziani M (2011) How inhibition shapes cortical activity. Neuron 72:231-243.

Ito HT, Zhang SJ, Witter MP, Moser EI, Moser MB (2015) A prefrontalthalamo-hippocampal circuit for goal-directed spatial navigation. Nature 522:50-55.

Jiang X, Shen S, Cadwell CR, Berens P, Sinz F, Ecker AS, Patel S, Tolias AS (2015) Principles of connectivity among morphologically defined cell types in adult neocortex. Science 350:aac9462.

Jones MW, Wilson MA (2005) Theta rhythms coordinate hippocampalprefrontal interactions in a spatial memory task. PLoS Biol 3:e402.

Kamigaki T, Dan Y (2017) Delay activity of specific prefrontal interneuron subtypes modulates memory-guided behavior. Nat Neurosci 20:854-863.

Katona I, Sperlágh B, Sík A, Käfalvi A, Vizi ES, Mackie K, Freund TF (1999) Presynaptically located CB1 cannabinoid receptors regulate GABA release from axon terminals of specific hippocampal interneurons. J Neurosci 19:4544-4558.

Kawaguchi Y, Kondo S (2002) Parvalbumin, somatostatin and cholecysto- kinin as chemical markers for specific GABAergic interneuron types in the rat frontal cortex. J Neurocytol 31:277-287.

Kawaguchi Y, Kubota Y (1997) GABAergic cell subtypes and their synaptic connections in rat frontal cortex. Cereb Cortex 7:476-486.

Kawaguchi Y, Kubota Y (1998) Neurochemical features and synaptic connections of large physiologically-identified GABAergic cells in the rat frontal cortex. Neuroscience 85:677-701.

Keimpema E, Straiker A, Mackie K, Harkany T, Hjerling-Leffler J (2012) Sticking out of the crowd: the molecular identity and development of cholecystokinin-containing basket cells. J Physiol 590:703-714.

Kepecs A, Fishell G (2014) Interneuron cell types are fit to function. Nature 505:318-326.

Kim D, Jeong H, Lee J, Ghim JW, Her ES, Lee SH, Jung MW (2016) Distinct roles of parvalbumin- and somatostatin-expressing interneurons in working memory. Neuron 92:902-915.

Kim EJ, Juavinett AL, Kyubwa EM, Jacobs MW, Callaway EM (2015) Three types of cortical layer 5 neurons that differ in brain-wide connectivity and function. Neuron 88:1253-1267.

Kim H, Ährlund-Richter S, Wang X, Deisseroth K, Carlén M (2016) Prefrontal parvalbumin neurons in control of attention. Cell 164:208-218.

Kim JC, Cook MN, Carey MR, Shen C, Regehr WG, Dymecki SM (2009) Linking genetically defined neurons to behavior through a broadly applicable silencing allele. Neuron 63:305-315.

Klausberger T, Somogyi P (2008) Neuronal diversity and temporal dynamics: the unity of hippocampal circuit operations. Science 321:53-57.

Kornblith S, Buschman TJ, Miller EK (2016) Stimulus load and oscillatory activity in higher cortex. Cereb Cortex 26:3772-3784.

Kubota Y, Kawaguchi Y (1997) Two distinct subgroups of cholecystokininimmunoreactive cortical interneurons. Brain Res 752:175-183.

Kupferschmidt DA, Gordon JA (2018) The dynamics of disordered dialogue: prefrontal, hippocampal and thalamic miscommunication underlying working memory deficits in schizophrenia. Brain Neurosci Adv 2:239821281877182.

Kuroda M, Yokofujita J, Oda S, Price JL (2004) Synaptic relationships between axon terminals from the mediodorsal thalamic nucleus and $\gamma$-aminobutyric acidergic cortical cells in the prelimbic cortex of the rat. J Comp Neurol 477:220-234.

Lagler M, Ozdemir AT, Lagoun S, Malagon-Vina H, Borhegyi Z, Hauer R, Jelem A, Klausberger T (2016) Divisions of identified parvalbuminexpressing basket cells during working memory-guided decision making. Neuron 91:1390-1401.

Lara AH, Wallis JD (2015) The role of prefrontal cortex in working memory: a mini review. Front Syst Neurosci 9:173.

Lee SY, Földy C, Szabadics J, Soltesz I (2011) Cell-type-specific CCK2 receptor signaling underlies the cholecystokinin-mediated selective excitation of hippocampal parvalbumin-positive fast-spiking basket cells. J Neurosci 31:10993-11002.

Lee S, Kruglikov I, Huang ZJ, Fishell G, Rudy B (2013) A disinhibitory circuit mediates motor integration in the somatosensory cortex. Nat Neurosci 16:1662-1670.

Lewis DA (2012) Cortical circuit dysfunction and cognitive deficits in schizophrenia: implications for preemptive interventions. Eur J Neurosci 35:1871-1878.

Lim L, Mi D, Llorca A, Marín O (2018) Development and functional diversification of cortical interneurons. Neuron 100:294-313.

Liu D, Gu X, Zhu J, Zhang X, Han Z, Yan W, Cheng Q, Hao J, Fan H, Hou R, Chen Z, Chen Y, Li CT (2014) Medial prefrontal activity during delay period contributes to learning of a working memory task. Science 346:458 - 463

Llano DA, Sherman SM (2009) Differences in intrinsic properties and local network connectivity of identified layer 5 and layer 6 adult mouse auditory corticothalamic neurons support a dual corticothalamic projection hypothesis. Cereb Cortex 19:2810-2826.

Lundqvist M, Rose J, Herman P, Brincat SL, Buschman TJ, Miller EK (2016) Gamma and beta bursts underlie working memory. Neuron 90:152-164.

Ma Y, Hu H, Berrebi AS, Mathers PH, Agmon A (2006) Distinct subtypes of somatostatin-containing neocortical interneurons revealed in transgenic mice. J Neurosci 26:5069-5082.

Marsicano G, Lutz B (1999) Expression of the cannabinoid receptor CB1 in distinct neuronal subpopulations in the adult mouse forebrain. Eur J Neurosci 11:4213-4225.

Marsman A, Mandl RC, Klomp DW, Bohlken MM, Boer VO, Andreychenko A, Cahn W, Kahn RS, Luijten PR, Hulshoff Pol HE (2014) GABA and 
glutamate in schizophrenia: a $7 \mathrm{~T} 1 \mathrm{H}-\mathrm{MRS}$ study. Neuroimage Clin 6:398-407.

Megías M, Emri Z, Freund TF, Gulyás AI (2001) Total number and distribution of inhibitory and excitatory synapses on hippocampal CA1 pyramidal cells. Neuroscience 102:527-540.

Miyoshi G, Hjerling-Leffler J, Karayannis T, Sousa VH, Butt SJ, Battiste J, Johnson JE, Machold RP, Fishell G (2010) Genetic fate mapping reveals that the caudal ganglionic eminence produces a large and diverse population of superficial cortical interneurons. J Neurosci 30:1582-1594.

Moghaddam B, Adams BW (1998) Reversal of phencyclidine effects by a group II metabotropic glutamate receptor agonist in rats. Science 281:1349-1352.

Moore TH, Zammit S, Lingford-Hughes A, Barnes TR, Jones PB, Burke M, Lewis G (2007) Cannabis use and risk of psychotic or affective mental health outcomes: a systematic review. Lancet 370:319-328.

Nigro MJ, Hashikawa-Yamasaki Y, Rudy B (2018) Diversity and connectivity of layer 5 somatostatin-expressing interneurons in the mouse barrel cortex. J Neurosci 38:1622-1633.

O'Neill PK, Gordon JA, Sigurdsson T (2013) Theta oscillations in the medial prefrontal cortex are modulated by spatial working memory and synchronize with the hippocampus through its ventral subregion. J Neurosci 33:14211-14224.

Parnaudeau S, O'Neill PK, Bolkan SS, Ward RD, Abbas AI, Roth BL, Balsam PD, Gordon JA, Kellendonk C (2013) Inhibition of mediodorsal thalamus disrupts thalamofrontal connectivity and cognition. Neuron 77:1151-1162.

Pawelzik H, Hughes DI, Thomson AM (2002) Physiological and morphological diversity of immunocytochemically defined parvalbumin- and cholecystokinin-positive interneurones in CA1 of the adult rat hippocampus. J Comp Neurol 443:346-367.

Pelkey KA, Chittajallu R, Craig MT, Tricoire L, Wester JC, McBain CJ (2017) Hippocampal GABAergic inhibitory interneurons. Physiol Rev 97:16191747.

Pfeffer CK, Xue M, He M, Huang ZJ, Scanziani M (2013) Inhibition of inhibition in visual cortex: the logic of connections between molecularly distinct interneurons. Nat Neurosci 16:1068-1076.

Pi HJ, Hangya B, Kvitsiani D, Sanders JI, Huang ZJ, Kepecs A (2013) Cortical interneurons that specialize in disinhibitory control. Nature 503:521-524.

Pinto L, Dan Y (2015) Cell-type-specific activity in prefrontal cortex during goal-directed behavior. Neuron 87:437-450.

Place R, Farovik A, Brockmann M, Eichenbaum H (2016) Bidirectional prefrontal-hippocampal interactions support context-guided memory. Nat Neurosci 19:992-994.

Postle BR, Druzgal TJ, D'Esposito M (2003) Seeking the neural substrates of visual working memory storage. Cortex 39:927-946.

Prescott SA (2014) Excitability: types I, II, and III. In: Encyclopedia of computational neuroscience, pp 1-7. New York: Springer.

Preston AR, Eichenbaum H (2013) Interplay of hippocampus and prefrontal cortex in memory. Curr Biol 23:R764-R773.

Prönneke A, Scheuer B, Wagener RJ, Möck M, Witte M, Staiger JF (2015) Characterizing VIP neurons in the barrel cortex of VIPcre/tdTomato mice reveals layer-specific differences. Cereb Cortex 25:4854-4868.

Ranganathan M, D'Souza DC (2006) The acute effects of cannabinoids on memory in humans: a review. Psychopharmacology (Berl) 188:425-444.

Riley MR, Constantinidis C (2016) Role of prefrontal persistent activity in working memory. Front Syst Neurosci 9:181.

Rodrigues LC, Conti CL, Nakamura-Palacios EM (2011) Clozapine and SCH 23390 prevent the spatial working memory disruption induced by $\Delta$ 9-THC administration into the medial prefrontal cortex. Brain Res 1382:230-237.

Rotaru DC, Barrionuevo G, Sesack SR (2005) Mediodorsal thalamic afferents to layer III of the rat prefrontal cortex: synaptic relationships to subclasses of interneurons. J Comp Neurol 490:220-238.

Roux F, Wibral M, Mohr HM, Singer W, Uhlhaas PJ (2012) Gamma-band activity in human prefrontal cortex codes for the number of relevant items maintained in working memory. J Neurosci 32:12411-12420.

Savanthrapadian S, Meyer T, Elgueta C, Booker SA, Vida I, Bartos M (2014)
Synaptic properties of SOM- and CCK-expressing cells in dentate gyrus interneuron networks. J Neurosci 34:8197-8209.

Schmitt LI, Wimmer RD, Nakajima M, Happ M, Mofakham S, Halassa MM (2017) Thalamic amplification of cortical connectivity sustains attentional control. Nature 545:219-223.

Sesack SR, Deutch AY, Roth RH, Bunney BS (1989) Topographical organization of the efferent projections of the medial prefrontal cortex in the rat: an anterograde tract-tracing study with phaseolus vulgaris leucoagglutinin. J Comp Neurol 290:213-242.

Sohal VS, Zhang F, Yizhar O, Deisseroth K (2009) Parvalbumin neurons and gamma rhythms enhance cortical circuit performance. Nature 459: $698-702$.

Somogyi J, Baude A, Omori Y, Shimizu H, El Mestikawy S, Fukaya M, Shigemoto R, Watanabe M, Somogyi P (2004) GABAergic basket cells expressing cholecystokinin contain vesicular glutamate transporter type 3 (VGLUT3) in their synaptic terminals in hippocampus and isocortex of the rat. Eur J Neurosci 19:552-569.

Spellman T, Rigotti M, Ahmari SE, Fusi S, Gogos JA, Gordon JA (2015) Hippocampal-prefrontal input supports spatial encoding in working memory. Nature 522:309-314.

Sreenivasan KK, Curtis CE, D’Esposito M (2014) Revisiting the role of persistent neural activity during working memory. Trends Cogn Sci 18:82-89.

Takács VT, Szonyi A, Freund TF, Nyiri G, Gulyás AI (2015) Quantitative ultrastructural analysis of basket and axo-axonic cell terminals in the mouse hippocampus. Brain Struct Funct 220:919-940.

Tamura M, Spellman TJ, Rosen AM, Gogos JA, Gordon JA (2017) Hippocampal-prefrontal theta-gamma coupling during performance of a spatial working memory task. Nat Commun 8:2182.

Taniguchi H (2014) Genetic dissection of GABAergic neural circuits in mouse neocortex. Front Cell Neurosci 8:8.

Tremblay R, Lee S, Rudy B (2016) GABAergic interneurons in the neocortex: from cellular properties to circuits. Neuron 91:260-292.

Tricoire L, Pelkey KA, Erkkila BE, Jeffries BW, Yuan X, McBain CJ (2011) A blueprint for the spatiotemporal origins of mouse hippocampal interneuron diversity. J Neurosci 31:10948-10970.

Tukker JJ, Fuentealba P, Hartwich K, Somogyi P, Klausberger T (2007) Cell type-specific tuning of hippocampal interneuron firing during gamma oscillations in vivo. J Neurosci 27:8184-8189.

van Aerde KI, Feldmeyer D (2015) Morphological and physiological characterization of pyramidal neuron subtypes in rat medial prefrontal cortex. Cereb Cortex 25:788-805.

Varvel SA, Lichtman AH (2002) Evaluation of CB1 receptor knockout mice in the Morris water maze. J Pharmacol Exp Ther 301:915-924.

Varvel SA, Hamm RJ, Martin BR, Lichtman AH (2001) Differential effects of $\triangle 9$-THC on spatial reference and working memory in mice. Psychopharmacology (Berl) 157:142-150.

Wang M, Yang Y, Wang CJ, Gamo NJ, Jin LE, Mazer JA, Morrison JH, Wang XJ, Arnsten AF (2013) NMDA receptors subserve persistent neuronal firing during working memory in dorsolateral prefrontal cortex. Neuron 77:736-749.

Wester JC, McBain CJ (2014) Behavioral state-dependent modulation of distinct interneuron subtypes and consequences for circuit function. Curr Opin Neurobiol 29:118-125.

Whissell PD, Cajanding JD, Fogel N, Kim JC (2015) Comparative density of CCK- and PV-GABA cells within the cortex and hippocampus. Front Neuroanat 9:124.

Wilson RI, Nicoll RA (2001) Endogenous cannabinoids mediate retrograde signalling at hippocampal synapses. Nature 410:588-592.

Xu H, Jeong HY, Tremblay R, Rudy B (2013) Neocortical somatostatinexpressing GABAergic interneurons disinhibit the thalamorecipient layer 4. Neuron 77:155-167.

Xu X, Roby KD, Callaway EM (2010) Immunochemical characterization of inhibitory mouse cortical neurons: three chemically distinct classes of inhibitory cells. J Comp Neurol 518:389-404.

Zhang S, Xu M, Chang WC, Ma C, Hoang Do JP, Jeong D, Lei T, Fan JL, Dan Y (2016) Organization of long-range inputs and outputs of frontal cortex for top-down control. Nat Neurosci 19:1733-1742. 